\title{
An additional update on the genus Chaetomium with descriptions of two coprophilous species, new to Italy
}

\author{
Doveri $\mathbf{F}^{1}$ \\ ${ }^{1}$ via dei Funaioli 22, I 57126 Livorno, Italy, f.doveri@sysnet.it
}

Doveri F 2013 - An additional update on the genus Chaetomium with descriptions of two coprophilous species, new to Italy. Mycosphere 4(4), 820-846, Doi 10.5943/mycosphere/4/4/17

\begin{abstract}
Two Chaetomium species new to Italy, C. brasiliense and C. succineum, are described and an updated key to coprophilous Chaetomium from Italy is provided. All author's collections of 23 species so far known from dung in Italy are listed. Following the recent publications describing new species, the worldwide key to Chaetomium species is updated.
\end{abstract}

Key words - dichotomous keys - dung - literature - records

\section{Introduction}

The present update follows Doveri $(2008,2011)$ and follows the monograph of Doveri (2004) on coprophilous ascomycetes and basidiomycetes from Italy. Doveri (2004) described and keyed out 13 Chaetomium species isolated from a variety of herbivore dung, and briefly redescribed 15 Chaetomium species published after von Arx et al. (1986) study of Chaetomium. Doveri (2008) provided an updated worldwide key to Chaetomium species, based on the monograph of von Arx et al. (1986), and described C. carinthiacum, C. gangligerum, $C$. homopilatum, C. spinosum, $C$. subaffine and $C$. variostiolatum as species new to Italy from dung. The species were illustrated with colour photographs. Doveri (2011) described C. murorum and $C$. ancistrocladum as new to Italy, and provided a brief update on the genus. Since Doveri (2011), seven new Chaetomium species have been introduced (Asgari \& Zare 2011, Ponsuriya et al. 2011). In the present work, the new species are placed in an updated worldwide key. C. succineum and $C$. brasiliense, isolated from dung are new records for Italy and full descriptions and illustrations are given. A key to 23 coprophilous species so far known from my Italy is also provided.

\section{Materials \& Methods}

The two Chaetomium species new to Italy were obtained from different types of dung cultured in non-axenic damp chambers, and studied according to Richardson \& Watling (1997) and Richardson (2001), slightly modified by Doveri (2004).

Abbreviations: CLSM = author's personal herbarium; d.c. = damp chamber culture; MCVE $=$ Herbarium of Venetian Civic Museum. 


\section{Taxonomy}

Chaetomium brasiliense Bat. \& Pontual, Bol. Agr. Com. Pernambuco 15: 701948.

Figs $1-10$

$\equiv$ Chaetomium hamatum Bat. \& Pontual, Bol. Agr. Com. Pernambuco 15: 701948.

$\equiv$ Chaetomium repandum Bat. \& Pontual, Bol. Agr. Com. Pernambuco 15: 701948.

三Chaetomium velutinum L.M. Ames, Mycologia 41: 6371949.

三Chaetomium alboarenulum Ames, U. S. Army Res. Develop., ser. 2: 161963.

इChaetomium congoense Ames, U. S. Army Res. Develop., ser. 2: 181963.

इChaetomium leucophora Ames, U. S. Army Res. Develop., ser. 2: 281963.

三Chaetomium perpulchrum Ames, U. S. Army Res. Develop., ser. 2: 331963.

(Synonyms $=$ fide von Arx et al. 1986)

Material examined - Italy, Tuscany, Livorno, Quercianella, $0 \mathrm{~m}$ a.s.1., about fifty scattered or gregarious, superficial specimens on fallow deer (Dama dama) dung in d.c., 22 Dec 2012, F. Doveri, CLSM 001.13.
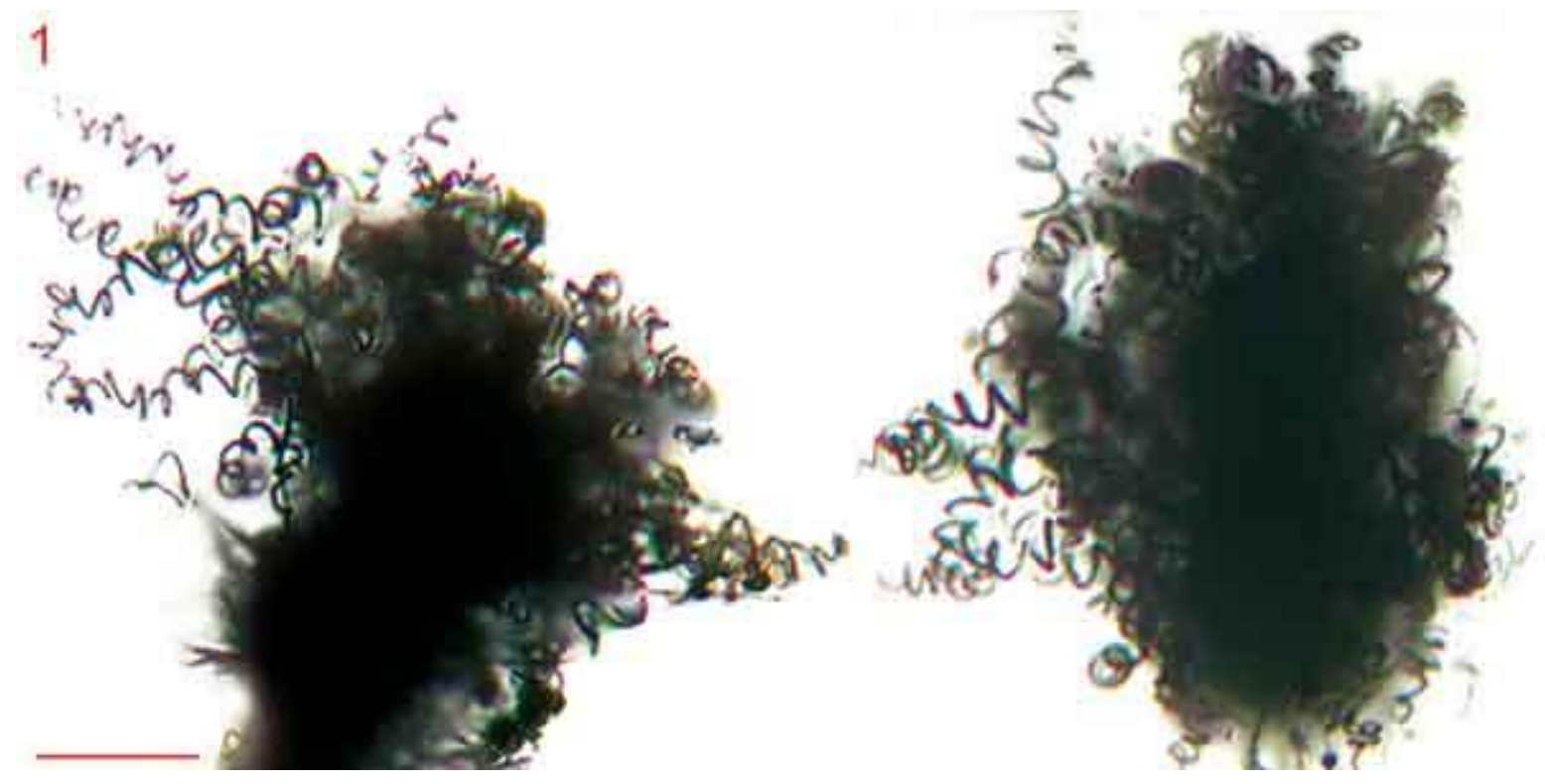

Fig. 1 - Chaetomium brasiliense. CLSM 001.13. Ascomata. - Bar $=100 \mu \mathrm{m}$.

Perithecia globose, subglobose or broadly ovoid, 100-220 $\mu \mathrm{m}$ diam., dark grey, membranous, entirely hairy with hairs denser around the ostiole. Peridium thin, pseudoparenchymatous, a textura angularis of dark brown, thick-walled, polygonal cells, 5-15 $\times$ 4-11 $\mu \mathrm{m}$. Terminal hairs straight at their base, long spirally coiled at the apex, without secondary coiled branches, up to $6 \mu \mathrm{m}$ diam. at the base, 4-6 $\mu \mathrm{m}$ diam. upwards, dark brown, strongly encrusted, thick-walled, densely septate, with roundish, slightly inflexed ends. Lateral and basal hairs somewhat narrower, up to $4 \mu \mathrm{m}$ diam., pale brown, enlarged at the base, septate, verruculose, attenuate towards the apex, straight or flexuous. Paraphyses not observed. Asci unitunicate, nonamyloid, cylindric, fasciculate, long-stalked, 60-70 × 7-8 $\mu \mathrm{m}$. Ascospores uniseriate, hyaline at first, dark grey at maturity, broadly ovoid in frontal view, bilaterally flattened and ellipsoidal or

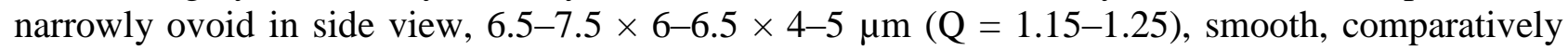
thin-walled, with an inconspicuous apiculus and a small, central germ pore at the pointed end. 


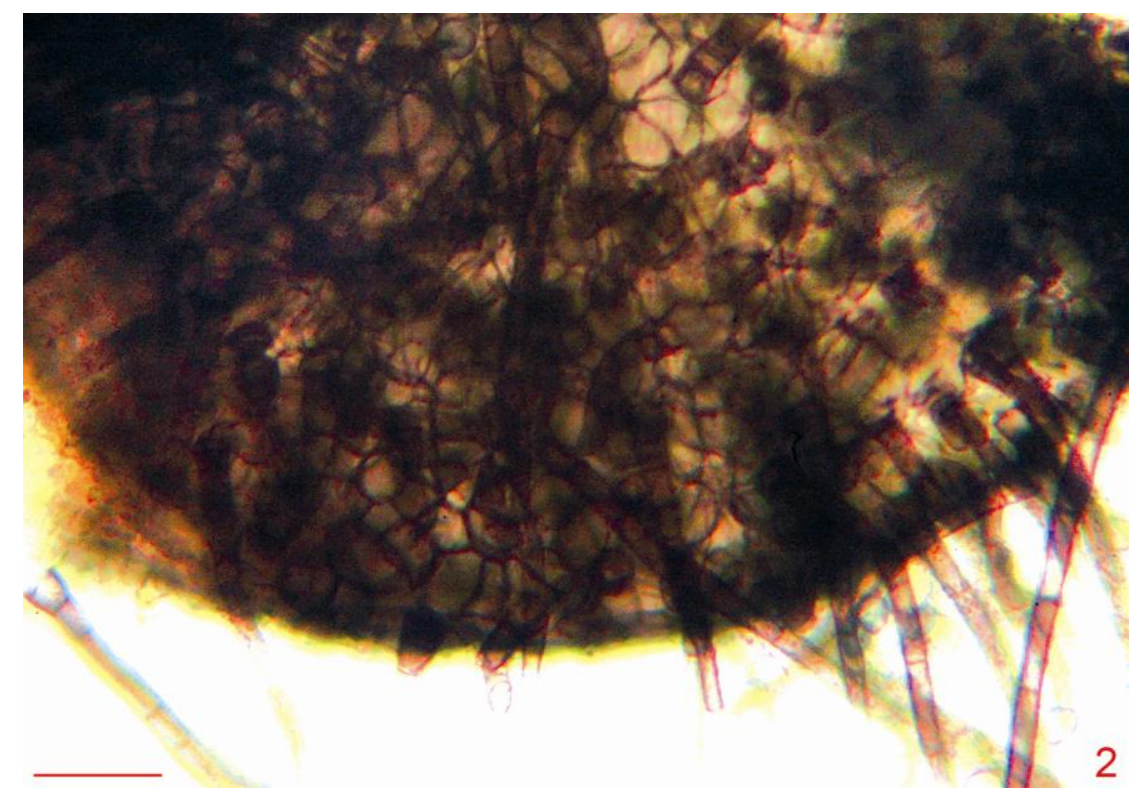

Fig. 2 - Chaetomium brasiliense. CLSM 001.13. Perithecial base. - Bar $=25 \mu \mathrm{m}$.

Notes $-C$. brasiliense is characterised by subglobose to ovate ascomata, undulate or spirally coiled terminal hairs, cylindrical asci, uniseriate, broadly ovoid, bilaterally flattened, comparatively small ascospores with an apical germ pore, and frequently grows on a variety of herbivore dung (Ames 1963, Lodha 1964, Singh \& Chanda Jain 1969, Saxena \& Mukerji 1973, Millner 1975, Liou \& Chen 1979, Lorenzo 1993, Doveri 2004, Bell 2005). It has also been isolated from seeds (Skolko \& Groves 1953), soil (Udagawa 1960) and textiles (Ames 1963). According to several records worldwide (Udagawa 1960, Seth 1970, von Arx et al. 1986) its spore size ranges between $6 \times 4 \mu \mathrm{m}$ and $8.5 \times 7.5 \mu \mathrm{m}$ (width in frontal view), whereas Lorenzo (1993) and Bell (2005) described it with larger ascospores $(8-10.5 \times 6-8 \mu \mathrm{m})$, more similar to those of $C$. mollicellum. The latter is hardly distinguishable from $C$. brasiliense, as having only slightly larger ascospores $(8-10 \times 5-7 \mu \mathrm{m}$ in the protologue, Ames 1963; 7-9.5 $\times 5-8 \mu \mathrm{m}$, Seth 1970, von Arx et al. 1986, Doveri 2004) and narrower (2-3 $\mu \mathrm{m}$ diam.) terminal hairs with a higher number of coils (von Arx et al. 1986). Also C. mollicellum has often been isolated from a variety of dung (Ames 1963, Lodha 1964, Carter \& Khan 1982, Doveri et al. 2000).

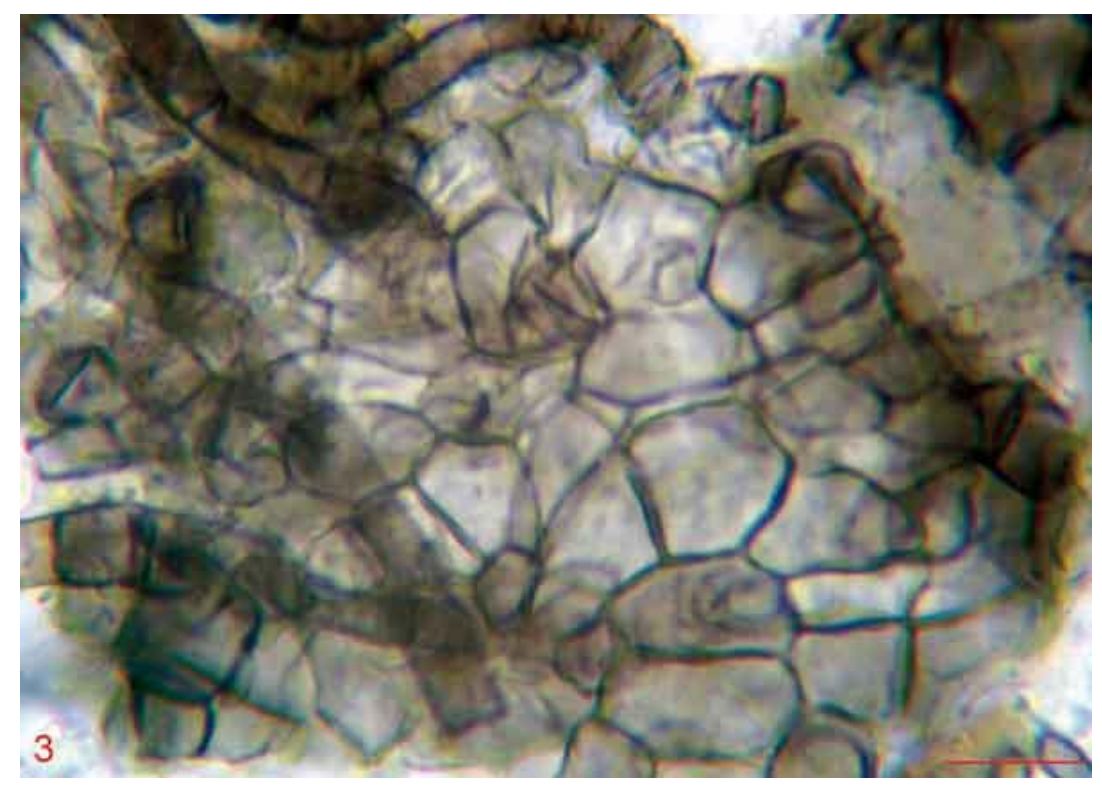

Fig. 3 -Chaetomium brasiliense. CLSM 001.13. Detail of peridial frame. - Bar $=10 \mu \mathrm{m}$. 
Chaetomium medusarum is similar to $C$. brasiliense and C. mollicellum, but differs from both in having thicker, 5-8 $\mu \mathrm{m}$ diam. (von Arx et al. 1986, Doveri 2004), spirally coiled terminal hairs with secondary and tertiary coiled branches (Meyer \& Lanneau 1967). It is a rare species but, like the other two, has often been isolated from dung (Carter \& Khan 1982, von Arx et al. 1986, Doveri et al. 2000).

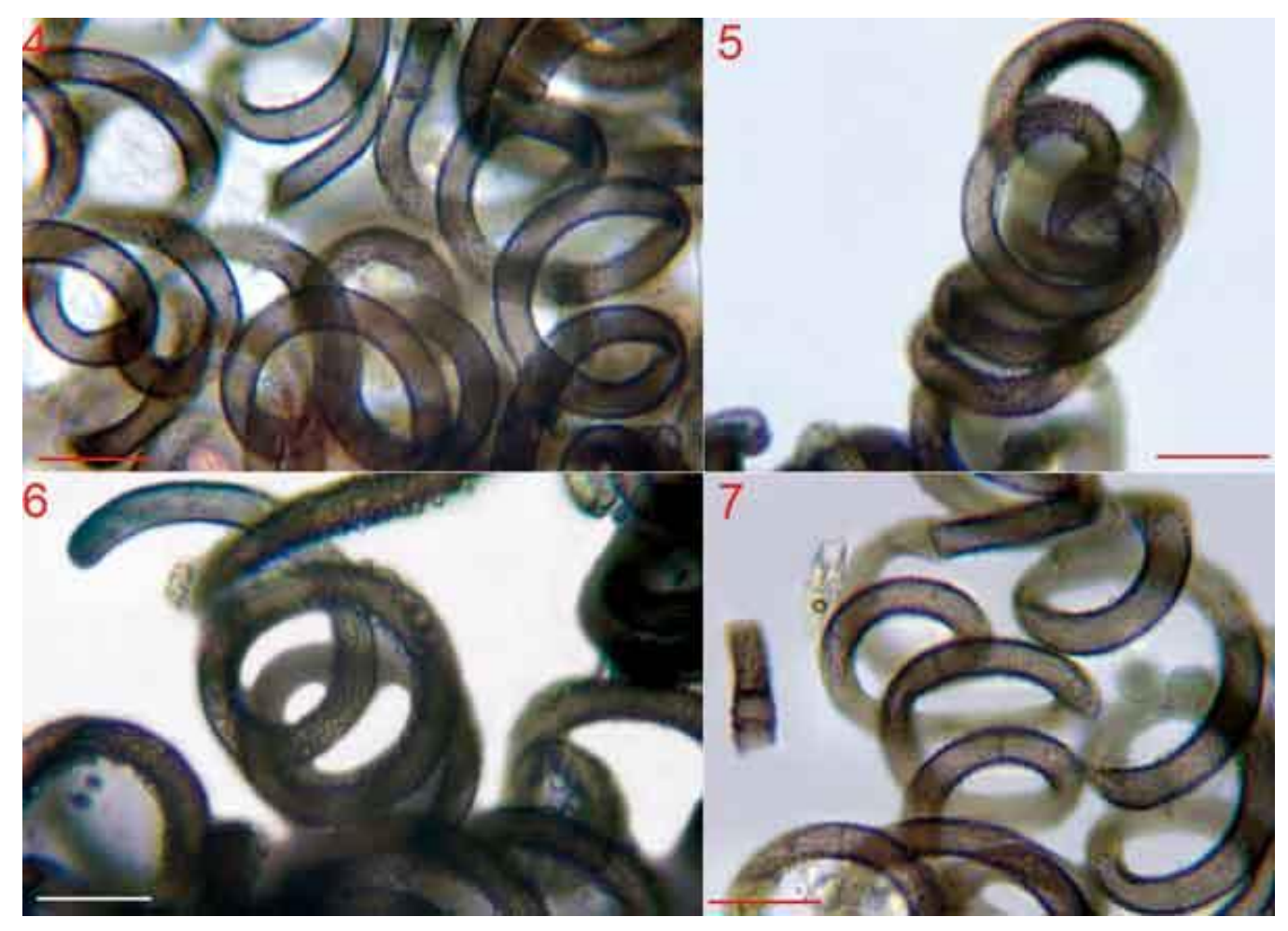

Figs 4-7 - Chaetomium brasiliense. CLSM 001.13. Terminal hairs. - Bars $=15 \mu \mathrm{m}$.

Chaetomium brasiliense has been subject of several biochemical studies and proved to produce secondary metabolites with important applications in agriculture and medicine. A chaetochalasin extracted from this fungus exhibits a cytotoxic activity, and some chaetoglobosins have an antifungal (Sibounnavong et al. 2012) and strong antiinsectan activity against Helicoverpa $z e a$, the agent of a major agricultural pest (Oh et al. 1998).

Further studies proved $C$. brasiliense to produce some mollicellins with growth inhibitory activity against human breast and lung cancer and neuroma (Li et al. 2008), and other mollicellins with similar cytotoxicity and an antimalarial activity (Khumkomkhet et al. 2009).

C. brasiliense has recently been identified as a new opportunistic agent of human otitis externa (Hubka et al. 2011).

Chaetomium succineum L.M. Ames, Mycologia 41: 4451949.

Figs $11-13$

Material examined - Italy, Tuscany, Livorno, Elba island, ? $\mathrm{m}$ a.s.l., one superficial specimen on sheep (Ovis aries) dung in d.c., 8 Apr 2011, L. Levorato, CLSM 003.12. 


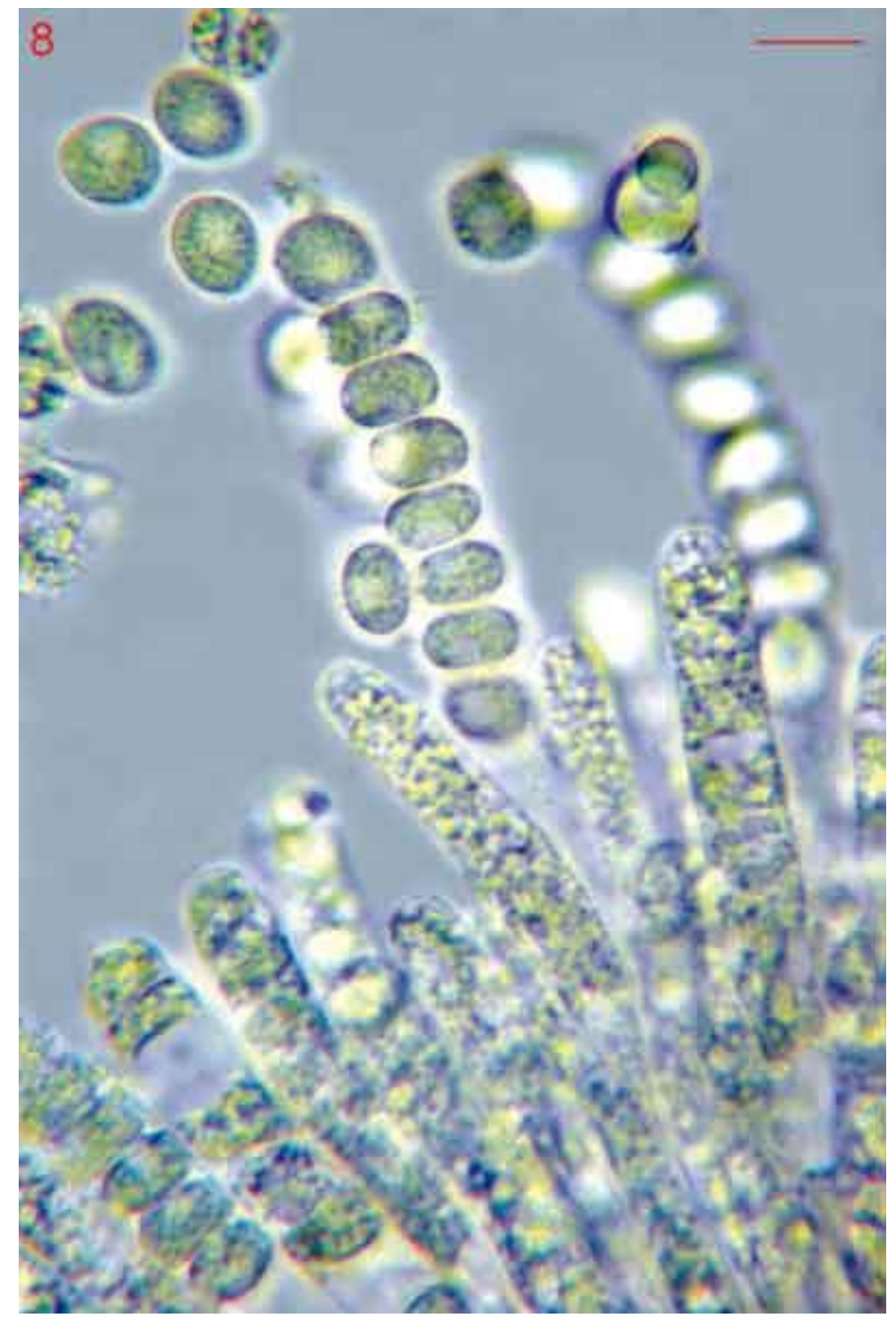

Fig. 8 - Chaetomium brasiliense. CLSM 001.13. Cylindrical asci with uniseriate ascospores. - Bar $=7 \mu \mathrm{m}$.

Perithecium $150 \mu \mathrm{m}$ diam., globose, ochraceous, membranous, entirely hairy with hairs particularly gathered around the almost inperceptible ostiole. Peridium very thin, with an outer layer of texura angularis formed of pale ochraceous, thin-walled, polygonal cells, 6-12 $\times 4-9 \mu \mathrm{m}$. Terminal hairs delicate, undulate to loosely spirally coiled, $2-2.5 \mu \mathrm{m}$ diam., up to $4 \mu \mathrm{m}$ at the base, verruculose or spinulose, pale brown, thin-walled, septate, somewhat rounded at the tips. Lateral hairs similar to the apical ones, but sparser. Paraphyses not observed. Asci unitunicate, nonamyloid, ephemeral, 60-75 × 13-15 $\mu \mathrm{m}, 8$-spored, clavate, long-stalked. Ascospores biseriate, hyaline in the early stages and containing several oil drops, becoming grey, finally greyish brown, (12-) 13-13.5 (-14) × (-7) 7.5-8 (-8.5) $\mu \mathrm{m}$, ellipsoidal $(\mathrm{Q}=1.50-1.92 ; \mathrm{Q}=1.67)$, often slightly inequilateral or even somewhat deformed, with attenuated, subacute ends and an apical germ pore, smooth, thin-walled.

Notes - C. succineum belongs to a group of species characterised by comparatively large ascomata (> $150 \mu \mathrm{m}$ diam.), clavate asci, medium-large (11-20 $\mu \mathrm{m}$ long), ellipsoid-fusiform ascospores with an usually apical germ pore, and absence of asexual state. 


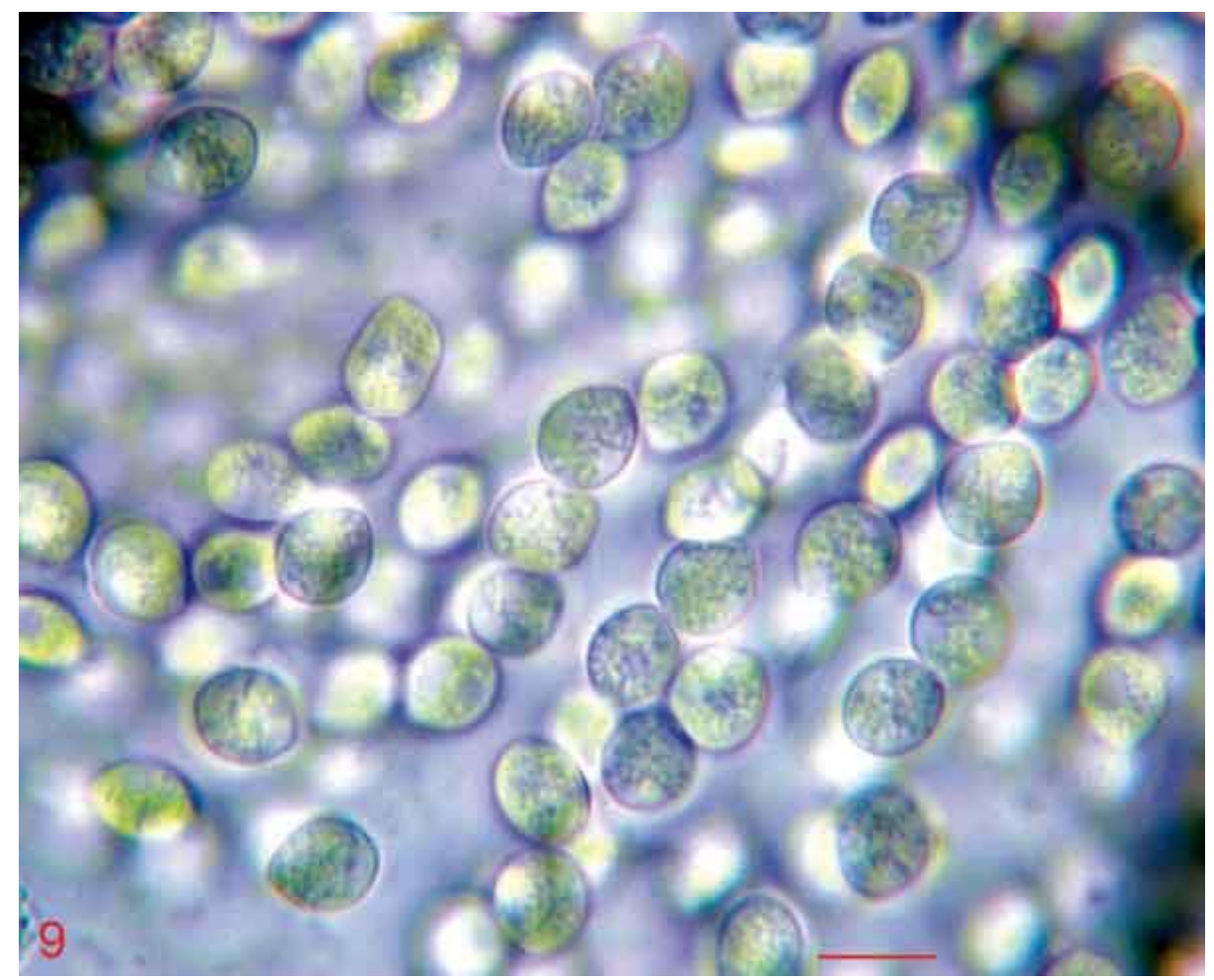

Fig. 9 - Chaetomium brasiliense. CLSM 001.13. Immature ascospores. - Bar $=8 \mu \mathrm{m}$.

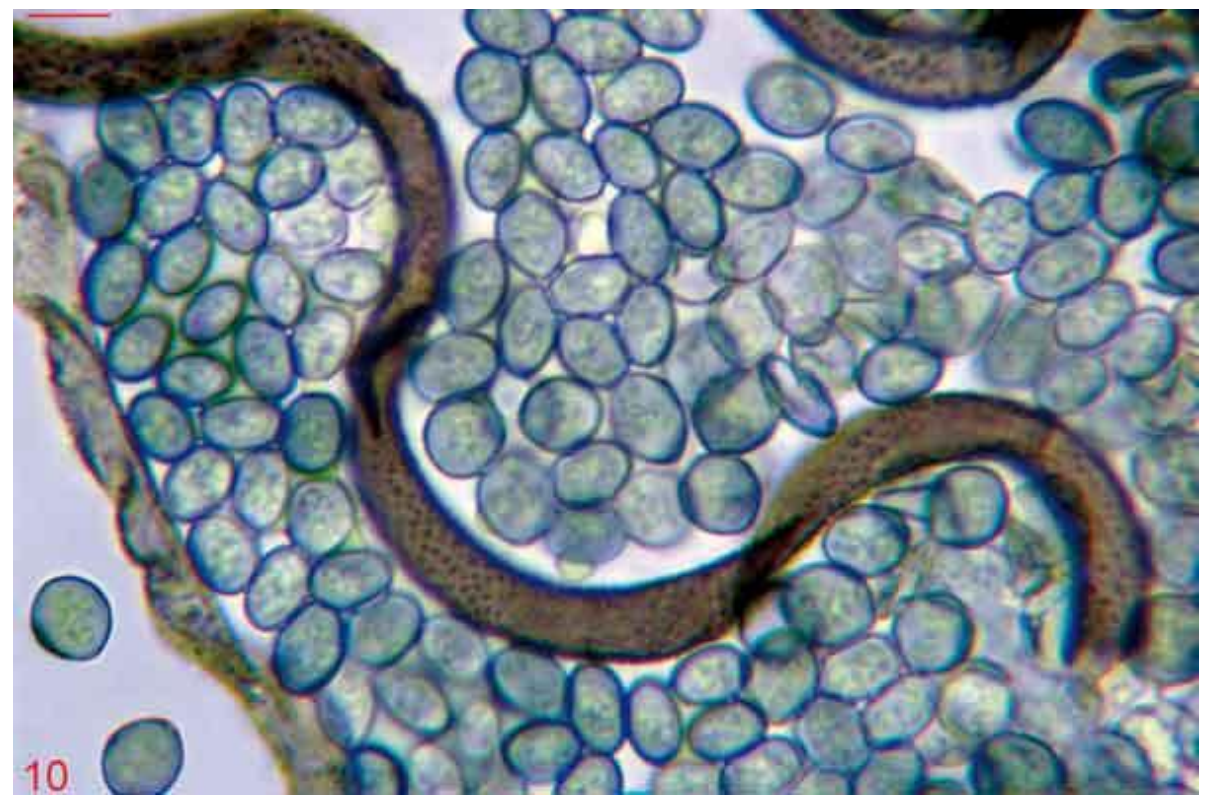

Fig. 10 - Chaetomium brasiliense. CLSM 001.13. Mature ascospores between undulate hairs. - Bar $=8 \mu \mathrm{m}$. 


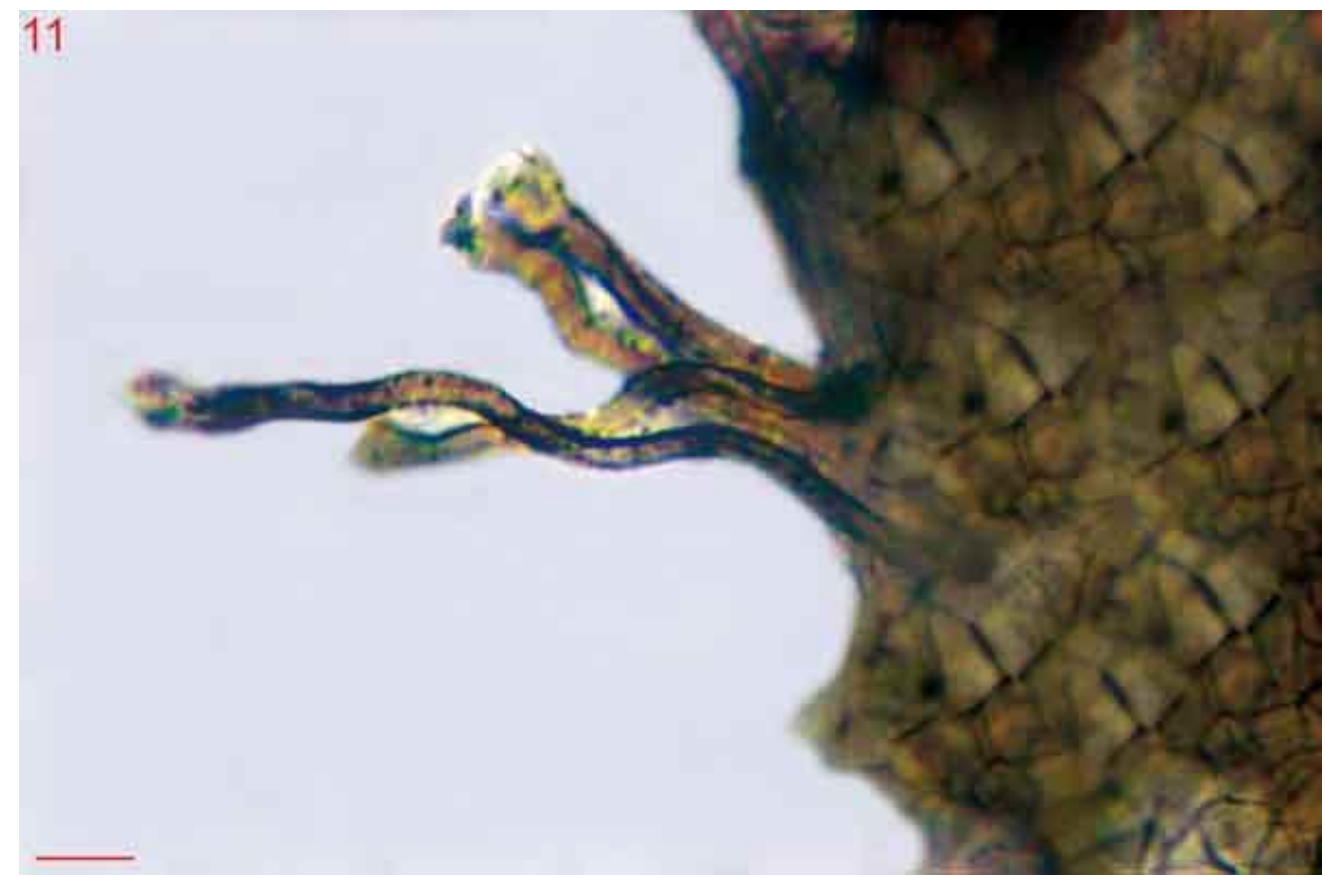

Fig. 11 - Chaetomium succineum. CLSM 003.12. Detail of peridium with undulate terminal hairs. - Bar $=7 \mu \mathrm{m}$.

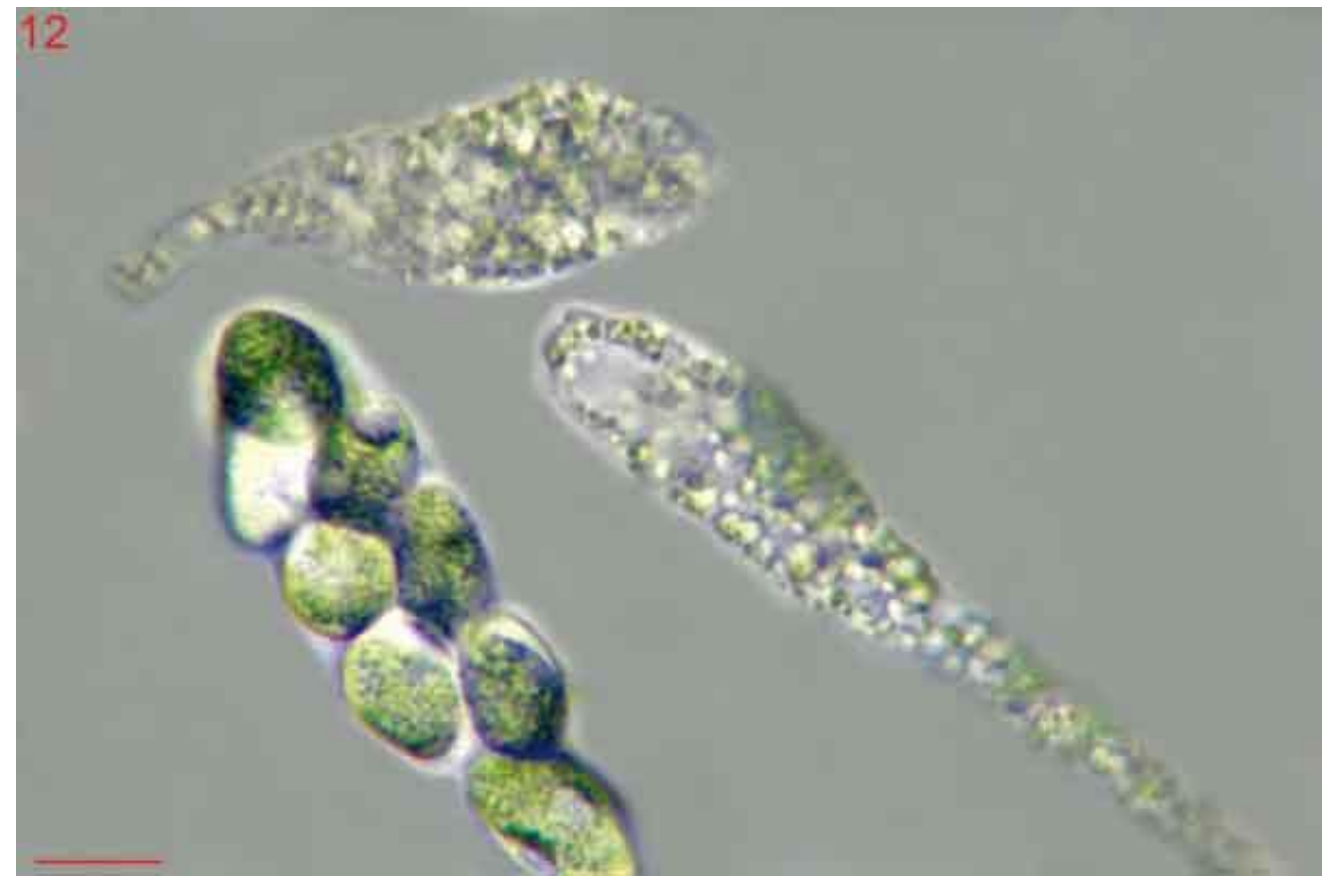

Fig. 12 - Chaetomium succineum. CLSM 003.12. Clavate asci. - Bar $=10 \mu \mathrm{m}$. 


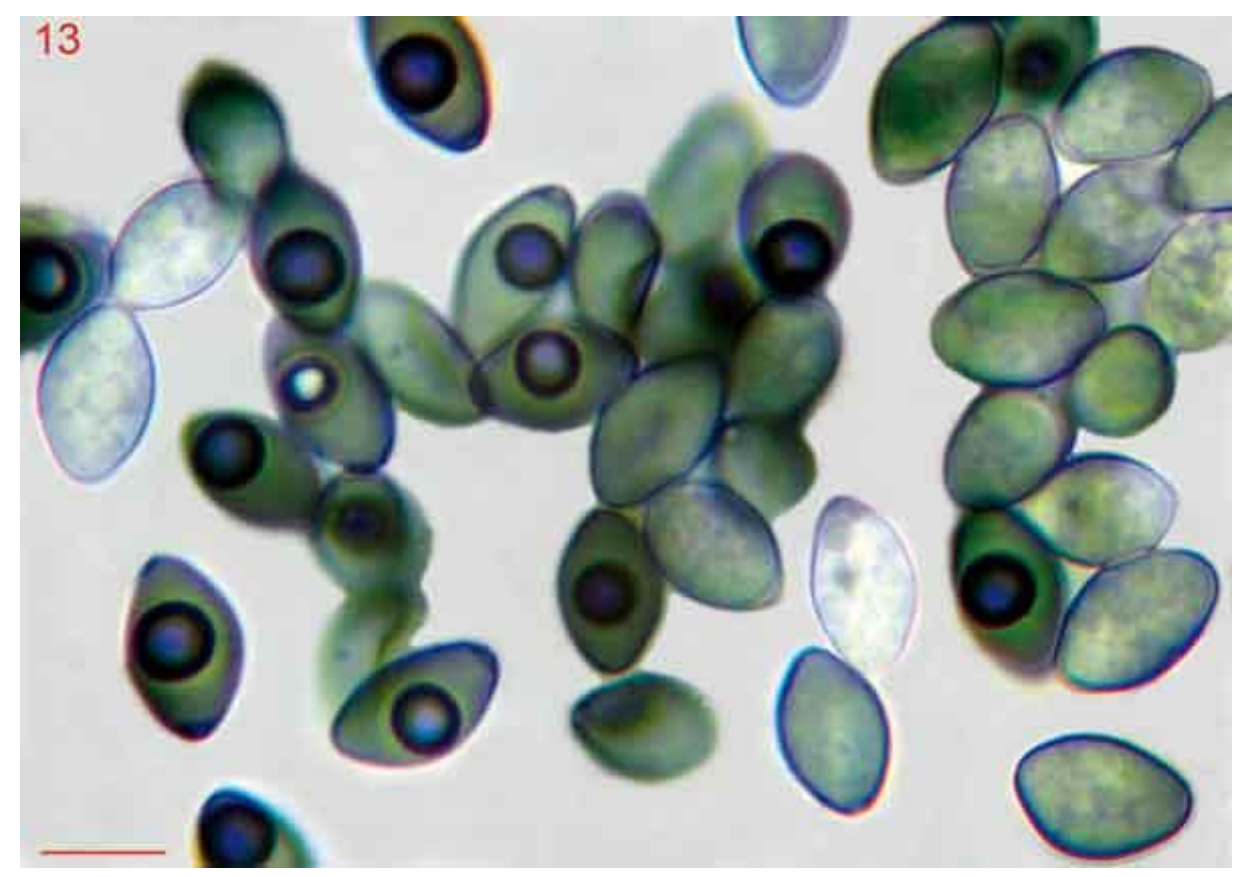

Fig. 13 - Chaetomium succineum. CLSM 003.12. Ascospores in different stages. - Bar $=8 \mu \mathrm{m}$.

Chaetomium succineum has been isolated from different substrata, e.g. soil (Pornsuriya et al. 2008), vegetable material (Ames 1949, 1963, Skolko \& Groves 1953, Chandra 1964) and often from a variety of dung (Carter \& Khan 1982, Lorenzo 1993, Bell 2005).

Chaetomium succineum was described by Pornsuriya et al. (2008) with ascospores 7.1-10 $\times$ 4.2-5.9 $\mu \mathrm{m}$, a size not matching either the protologue (Ames 1949, 12-15 $\times 7-8 \mu \mathrm{m}$ ) or subsequent descriptions (Skolko \& Groves 1953, Udagawa 1960, Seth 1970, von Arx et al. 1986). Chandra (1964) recorded a collection of $C$. succineum infecting leaves of Cannabis sativa L. with globose to ovate ascospores, even reniform in the linked drawing, not corresponding to the protologue. Ames (1949) really mentioned "globose-ovate" ascospores, "rounded to subacute at the ends", but she drew, at that time and later (1963), ellipsoid-fusiform ascospores, like those defined and photographed by von Arx et al. (1986), who revised the type strain.

Although $C$. succineum is known not to produce exudates in culture, Bell (2005) described an Australian collection producing yellow exudates on cornmeal agar.

Chaetomium succineum is similar to $C$. murorum which differs, however, in having flexuous to circinate, rather than loosely spiralate, terminal hairs, a peridium with a variable textura also in the same ascoma (Doveri 2011), and ascospores usually with some darker longitudinal bands (von Arx et al. 1986). Also C. murorum has been recorded from a variety of dung (Karsten 1888, Massee \& Salmon 1901, 1902, Bainier 1909, Palliser 1910, Stratton 1921, Skolko \& Groves 1953, Ames 1963, Tóth 1963, 1965, 1967, Mirza \& Nasir 1968, Seth 1968, 1970, Calviello 1971, Ahmad \& Sultana 1973, Hubálek 1974, Matsushima 1975, Millner 1975, Carter \& Khan 1982, Barrasa 1985, Bokhary \& Parvez 1986, Valldosera 1991, Eriksson 1992, Caretta et al. 1994, 1998, Caretta \& Piontelli 1996, Beyer 1999, Richardson 2004, Elshafie 2005, Piontelli et al. 2006, Chang \& Wang 2009, Doveri 2011), although it has a preference for leporine droppings (Mirza \& Nasir 1968, Seth 1968, Piontelli et al. 2006, Favre 2008).

According to von Arx et al. (1986) C. subspirilliferum Sergeeva is also similar to $C$. succineum, but is distinguishable in having much smaller ascomata. We have not found, in the original descriptions, other significant differences between the two species.

\section{Update on collections of coprophilous species from Italy}

(for synonyms, see von Arx et al. 1986 and Doveri 2008) 
Chaetomium ancistrocladum Udagawa \& Cain, Can. J. Bot. 47: 19431969.

MATERIAL: 1) VICENZA, Longare, Mount Brosimo, $100 \mathrm{~m}$, on dormouse dung in d.c., A. Bizzi, 6.6.11, 125.2-Longare CLSM 009.08. 2) VICENZA, Longare-Mount Brosimo, $100 \mathrm{~m}$, on dormouse dung in d.c., A. Bizzi, 2.6.10, 125.2-Longare, CLSM 009.08-bis.

TOTAL 2: dormouse.

Chaetomium bostrychodes Zopf, Abh. Bot. Ver. Prov. Brandenb. 19: 1731887.

MATERIAL: 1) VENEZIA, Caroman, $0 \mathrm{~m}$, dozens of gregarious specimens on wild rabbit dung in d.c., F. Doveri, 5.97, 148.2-Chioggia, MCVE 667. 2) VENEZIA, Alberoni, 0 m, on wild rabbit dung, E. Bizio, 5.97, 148.1-Alberoni, CLSM 02197 bis. 3) FERRARA, Mesola, 0 m, on badger dung in d.c., F. Bersan \& G. Visentin, 5.97, 187.1-Mesola, CLSM 02197 ter. 4) TREVISO, Scalon, $200 \mathrm{~m}$, on sheep dung in d.c., 6.6.97, 083.1-Valdobbiadene, CLSM 02197 quater. 5) LIVORNO, Quercianella, 0 m, on toad dung in d.c., C. Doveri, 10.97, 284.3-Rosignano Marittimo, CLSM 02197 penta. 6) COSENZA, Fossiata, 1400 m, on lizard dung in d.c., C. Lavorato, 22.5.98, 560.1-Mount Volpintesta, CLSM 02197 esa. 7) GROSSETO, Principina terra, 0 m, on rabbit dung in d.c., F. Doveri, 331.3-Alberese, CLSM 02197 epta. 8) GROSSETO, Principina terra, 0 m, on wild pig dung in d.c., F. Doveri, 331.3-Alberese, CLSM 02197 octo. 9) GROSSETO, Punta Ala, 0 $\mathrm{m}$, on hedgehog dung in d.c., F. Doveri and F. Bersan, 2.4.98, 318.3-Punta Ala, CLSM 02197 ena. 10) UDINE, Sauris di Sopra, $1400 \mathrm{~m}$, on horse dung in d.c., G. Medardi, 25.6.98, 030.2-Forni di Sopra, CLSM 02197 deca. 11) COSENZA, Corigliano Calabro (Contrada Tenimento), $300 \mathrm{~m}$, on pigeon dung in d.c., C. Lavorato, 16.5.98, 552.1-Corigliano Calabro, CLSM 02197-XI. 12) COSENZA, Corigliano Calabro (Contrada Tenimento), $300 \mathrm{~m}$, on goat dung in d.c., C. Lavorato, 16.5.98, 552.1-Corigliano Calabro, CLSM 02197-XII. 13) LIVORNO, 0 m, on snail dung in d.c., F. Doveri, 20.9.98, 283.1-Livorno, CLSM 02197-XIII. 14) COSENZA, S. Benedetto Acri, 700 m, on hedgehog dung in d.c., C. Lavorato, 7.8.98, 552.4-S. Demetrio Corone, CLSM 02197-XIV. 15) COSENZA, S. Demetrio Corone (Contrada Poggio), $600 \mathrm{~m}$, on sparrow dung in d.c., C. Lavorato, 26.5.98, 552.4-S. Demetrio Corone, CLSM 02197-XV. 16) BELLUNO, Canale d'Agordo (loc. Pisoliva), $1100 \mathrm{~m}$, on hare dung in d.c., E. Bizio, 14.6.98, 046.3-Agordo, CLSM 02197-XVI. 17) VENEZIA, Carpenedo wood, 0 m, on dung of unidentified bird, G. Robich, 26.6.99, 127.2-Mestre, CLSM 02197-XVII. 18) BRESCIA, Tonale Pass, $2200 \mathrm{~m}$, on roe deer dung, F. Bersan, 8.9.99, 041.2-Ponte di Legno, CLSM 02197-XVIII. 19) BELLUNO, Forcella Negher, $2360 \mathrm{~m}$, on chamois dung in d.c., E. Bizio, 6.9.99, 045.1-Cencenighe Agordino, CLSM 02197-XIX. 20) MODENA, Zocca, $750 \mathrm{~m}$, on hedgehog dung in d.c., L. Piccioli, 12.99, 237.4-Savigno, CLSM 02197-XX. 21) NUORO, Tertenia (loc. Sucrabiolus), $150 \mathrm{~m}$, on sheep dung in d.c., A. Bizzi, 7.11.99, 541.2Tertenia, CLSM 02197-XXI. 22) COSENZA, S. Demetrio Corone (Contrada Calamia), 800 m, on house mouse dung in d.c., C. Lavorato, 16.8.99, 552.4-S. Demetrio Corone, CLSM 02197-XXII. 23) CAGLIARI, Villasimíus, $100 \mathrm{~m}$, on sheep dung in d.c., A. Bizzi, 9.11.99, 567.1-Villasimíus, CLSM 02197-XXIII. 24) NUORO, Cardedu (loc. Perda Pera), 0 m, on sheep dung in d.c., A. Bizzi, 7.11.99, 541.1-Jerzu, CLSM 02197-XXIV. 25) COSENZA, Serra del Prete, $1700 \mathrm{~m}$, on wild pig dung in d.c., C. Lavorato, 15.5.99, 534.4-Rotonda, CLSM 02197-XXV. 26) COSENZA, S. Demetrio Corone, $800 \mathrm{~m}$, on rat dung in d.c., C. Lavorato, 29.2.00, 552.4-S. Demetrio Corone, CLSM 02197-XXVI. 27) COSENZA, S. Demetrio Corone, $800 \mathrm{~m}$, on sparrow dung in d.c., C. Lavorato, 16.1.00, 552.4-S. Demetrio Corone, CLSM 02197-XXVII. 28) PISA, S. Rossore Park, 0 $\mathrm{m}$, on wild rabbit dung in d.c., F. Doveri, 5.3.00, 272.2-Migliarino, CLSM 02197-XXVIII. 29) LIVORNO, Corbolone, $150 \mathrm{~m}$, on horse dung in d.c., G. Cacialli \& F. Doveri, 14.4.00, 284.4Collesalvetti, CLSM 02197-XXIX. 30) MILANO, Triuggio, on wild rabbit dung in d.c., F. Doveri, 26.5.00, 096.2-Seregno, CLSM 02197-XXX. 31) MILANO, Monza Park, 150 m, on wild rabbit dung in d.c., F. Doveri, 26.5.00, 118.1-Monza, CLSM 02197-XXXI. 32) VENEZIA, Campalto, 0 $\mathrm{m}$, on toad (?) dung in d.c., G. Robich, 21.6.00, 127.2-Mestre, CLSM 02197-XXXII. 33) VICENZA, Alte Ceccato, on rat dung in d.c., A. Bizzi, 22.2.01, 125.3-Mountbello, CLSM 02197- 
XXXIII. 34) COSENZA, Rossano (Cozzo del Pesco), $900 \mathrm{~m}$, on sheep dung in d.c., C. Lavorato, 22.9.00, 552.1-Corigliano Calabro, CLSM 02197-XXXIV. 35) COSENZA, Rossano (Cozzo del Pesco), $900 \mathrm{~m}$, on hooded crow dung in d.c., C. Lavorato, 22.9.00, 552.1-Corigliano Calabro, CLSM 02197-XXXV. 36) COSENZA, S. Demetrio Corone (Contrada Calamia), $800 \mathrm{~m}$, on lizard dung in d.c., C. Lavorato, 19.8.01, 552.4-S. Demetrio Corone, CLSM 02197-XXXVI. 37) COSENZA, loc. Pettinascura-S. Giovanni in Fiore, $1600 \mathrm{~m}$, on beech-marten dung in d.c., C. Lavorato, 26.2.01, 560.1-Monte Volpintesta, CLSM 02197-XXXVII. 38) COSENZA, S. Demetrio Corone (Contrada Calamia), $800 \mathrm{~m}$, on squirrel dung in d.c., C. Lavorato, 10.7.01, 552.4-S. Demetrio Corone, CLSM 02197-XXXVIII. 39) COSENZA, S. Demetrio Corone (Contrada Calamia), $800 \mathrm{~m}$, on weasel dung in d.c., C. Lavorato, 16.8.01, 552.4-S. Demetrio Corone, CLSM 02197-XXXIX. 40) COSENZA, loc. Pettinascura-Longobucco, $1580 \mathrm{~m}$, on sheep dung in d.c., C. Lavorato, 2.4.01, 560.1-Monte Volpintesta, CLSM 02197-XL. 41) COSENZA, Manche di GrecaAcri, $1100 \mathrm{~m}$, on goat dung in d.c., C. Lavorato, 15.2.01, 552.3-Acri, CLSM 02197-XLI. 42) LECCE, Pisignano-Vernole, $40 \mathrm{~m}$, on sheep dung in d.c., V. Sciurti \& F. Doveri, 24.11.01, 512.1Lecce, CLSM 02197-XLII. 43) LECCE, Punta Prosciutto, 0 m, on sheep dung in d.c., P. Franchi \& M. Marchetti, 24.11.01, 511.3-Torre Colimena, CLSM 02197-XLIII. 44) LECCE, Vanze, 10 m, on goat dung in d.c., V. Sciurti \& F. Doveri, 24.11.01, 513.4-S. Foca, CLSM 02197-XLIV. 45) LECCE, Le Cesine wood (S. Foca), $0 \mathrm{~m}$, on fox (?) dung in d.c., F. Doveri, 22.11.01, 513.4-S. Foca, CLSM 02197-XLV. 46) ROMA, Castel Porziano-loc. Tor Paterno, $0 \mathrm{~m}$, on wild pig dung in d.c., G. Robich, 3.12.01, 386.1-Lido di Ostia, CLSM 02197-XLVI. 47) LECCE, Prosciutto point, 0 $\mathrm{m}$, on fox (?) dung in d.c., P. Franchi \& M. Marchetti, 24.11.01, 511.3-Torre Colimena, CLSM 02197-XLVII. 48) COSENZA, Corigliano Calabro-loc. Tenimento, $250 \mathrm{~m}$, on lizard dung in d.c., C. Lavorato, 16.11.01, 552.1-Corigliano Calabro, CLSM 02197-XLVIII. 49) COSENZA, Corigliano Calabro-loc. Tenimento, $250 \mathrm{~m}$, on pigeon dung in d.c., C. Lavorato, 16.11.01, 552.1Corigliano Calabro, CLSM 02197-XLIX. 50) COSENZA, Corigliano Calabro-loc. Tenimento, 250 $\mathrm{m}$, on hen dung in d.c., C. Lavorato, 16.11.01, 552.1-Corigliano Calabro, CLSM 02197-L. 51) COSENZA, Corigliano Calabro-loc. Tenimento, $250 \mathrm{~m}$, on hen dung in d.c., C. Lavorato, 23.10.01, 552.1-Corigliano Calabro, CLSM 02197-LI. 52) COSENZA, Acri-Contrada Mischinella, $1100 \mathrm{~m}$, on wild pig dung in d.c., C. Lavorato, 19.10.01, 552.3-Acri, CLSM 02197-LII. 53) COSENZA, S. Demetrio Corone, $800 \mathrm{~m}$, on polecat dung in d.c., C. Lavorato, 14.3.02, 552.4-S. Demetrio Corone, CLSM 02197-LIII. 54) COSENZA, Acri, $1050 \mathrm{~m}$, on beech-marten dung in d.c., C. Lavorato, 30.4.02, 552.3-Acri, CLSM 02197-LIV. 55) COSENZA, S. Sofia dell'Epiro, $600 \mathrm{~m}$, on tortoise dung in d.c., C. Lavorato, 28.8.02, 552.4-S. Demetrio Corone, CLSM 02197-LV. 56) VICENZA, Nogarole-Mount Faldo, 700 m, on hare dung in d.c., A. Bizzi, 20.4.04, CLSM 02197-LVI. 57) CAMPOBASSO, S. Massimo, 630 m, on roe deer dung in d.c., A. Bizzi, 14.10.04, 405.3-Boiano, CLSM 02197-LVII. 58) ROMA, Grotta di Piastra-Tenuta di Castel Porziano, Om, on bird dung, G. Robich, 24.11.04, 386.1-Lido di Ostia, CLSM 02197-LVIII. 59) GROSSETO, Follonica, 0 m, on fallow-deer dung in d.c., F. Doveri, 15.5.05, 318.4-Follonica, CLSM 02197-LIX. 60) COSENZA, S. Sofia d'Epiro, $250 \mathrm{~m}$, on porcupine dung in d.c., C. Lavorato, 12.7.05, 552.4-S. Demetrio Corone, CLSM 02197-LX. 61) LIVORNO, Palazzi di Cecina, $0 \mathrm{~m}$, on cattle dung in d.c., F. Doveri, 30.4.06, 294.1-Cecina, CLSM 02197-LXI. 62) VENEZIA, Chioggia-Bosco Nordio, 0 m, on fallow deer dung in d.c., L. Levorato, 8.4.06, 148.2-Chioggia, CLSM 02197-LXII. 63) FERRARA, Mesola-S. Giustina, 0 m, on roe deer dung in d.c., A. Bizzi, 10.11.05, 187.1-Mesola, CLSM 02197- LXIII. 64) VICENZA, Valdagno-Passo Zovo, $600 \mathrm{~m}$, on horse dung in d.c., A. Bizzi, 18.5.06, 102.2-Valdagno, CLSM 02197-LXIV. 65) VICENZA, Val Maron, $1400 \mathrm{~m}$, on hare dung in d.c., L. Levorato, 22.4.07, 083.4-Arsié, CLSM 02197-LXV. 66) VENEZIA, Caorle, Ca Corniani, 0 m, on horse dung in d.c., A. Bizzi, 27.10.07, 107.2-Ca Corniani, CLSM 02197-LXVI. 67) VENEZIA, Caorle, Brussa, $0 \mathrm{~m}$, on hare dung in d.c., A. Bizzi, 27.10.07, 107.2-Ca Corniani, CLSM 02197-LXVII. 68) VICENZA, Longare, Mount Brosimo, $100 \mathrm{~m}$, on dormouse dung in d.c., A. Bizzi, 6.6.11, 125.2-Longare, CLSM 02197-LXVIII. 69) VICENZA, Mossano, Mount Stria, 375 $\mathrm{m}$, on hedgehog dung in d.c., A. Bizzi, 21.7.08, 125.2-Longare, CLSM 02197-LXIX. 70) LIVORNO, Montioni, $0 \mathrm{~m}$, on sheep dung in d.c., F. Doveri, 18.3.09, 306.3-Suvereto, CLSM 
02197-LXX. 71) LIVORNO, Montioni, $0 \mathrm{~m}$, on rabbit dung in d.c., F. Doveri, 18.3.09, 306.3Suvereto, CLSM 02197-LXXI. 72) LIVORNO, Elba island, Campo nell'Elba, Prataccio loc. Valle Buia, $200 \mathrm{~m}$, on wild pig dung in d.c., C. Cotta, 10.2.11, 328.1-Marina di Campo, CLSM 02197LXXII. 73) PISA, Monteverdi Marittimo, podere Allumiere, $200 \mathrm{~m}$, on rabbit dung in d.c., F. Doveri, 25.3.11, 306.4-Monteverdi Marittimo, CLSM 02197-LXXIII. 74) PISA, La Sassa, 350 m, on roe deer dung in d.c., F. Doveri, 26.10.12, 295.3-Sassa, CLSM 02197-LXXIV. 75) LIVORNO, Quercianella, $50 \mathrm{~m}$, on roe deer dung in d.c., F. Doveri, 23.12.12, 284.3-Rosignano Marittimo, CLSM 02197-LXXV. 76) VENEZIA, Fusina, Cassa colmata A, 0 m, on hare dung in d.c., L. Levorato, 2.6.11, 127.2-Mestre, CLSM 02197-LXXVI.

TOTAL 76: sheep 9; hare 5; roe deer 5; wild pig 5; wild rabbit 5; hedgehog 4; horse 4; goat 3; lizard 3; rabbit 3; beech-marten 2; fallow deer 2; fox 2; hen 2; pigeon 2; rat 2; sparrow 2; toad 2; unidentified bird 2; badger 1; cattle 1; chamois 1 ; dormouse 1 ; hooded crow 1; mouse 1; polecat 1; porcupine 1; snail 1; squirrel 1; tortoise 1; weasel 1 .

\section{Chaetomium brasiliense}

MATERIAL: LIVORNO, Quercianella, $0 \mathrm{~m}$, about fifty scattered or gregarious, superficial specimens on fallow deer dung in d.c., F. Doveri, 22.12.2012, 284.3-Rosignano Marittimo, CLSM 001.13 .

TOTAL 1: fallow deer

Chaetomium carinthiacum Sörgel, Arch. Mikrobiol. 40: 3931961.

MATERIAL: ROMA, Castel Porziano estate-loc. Grotta di Piastra, $0 \mathrm{~m}$, five gregarious, superficial specimens in d.c., growing out from the dung of an unidentified bird, on to the blotting paper spotted with a yellow-green exudate, G. Robich, 24.11.04, 386.1-Lido di Ostia, CLSM 009.05 .

TOTAL 1: unidentified bird.

Chaetomium crispatum (Fuckel) Fuckel, Symb. Mycol.: 901870.

MATERIAL: VICENZA, Posina, $\mathrm{m}$ ?, four isolated specimens on roe deer dung in d.c., A. Bizzi, 25.5.02, 081.2-Castana, CLSM 020.02.

TOTAL 1: roe deer.

Chaetomium cuniculorum Fuckel, Fungi rhenani exsic. nº 19611867.

MATERIAL: 1) VENEZIA, Caroman, $0 \mathrm{~m}$, dozens of gregarious, superficial specimens, on wild rabbit dung in d.c., F. Doveri, 5.97, 148.2-Chioggia, MCVE 668. 2) CATANIA, Etna Park, $1200 \mathrm{~m}$, on wild rabbit dung in d.c., G. Robich, 9.11.98, 612.2-Randazzo, CLSM 02297 bis. 3) VENEZIA, Caorle-loc. Brussa, on wild rabbit dung in d.c., A. Bizzi, 10.10.04, 129.1-Caorle, CLSM 02297 ter. 4) FIRENZE, Nipozzano, 500 m, on hare dung in d.c., F. Doveri, 4.5.08, 276.4Pontassieve, CLSM 02297-quater. 5) FIRENZE, Nipozzano, 500 m, on roe deer (?) dung in d.c., F. Doveri, 4.5.08, 276.4-Pontassieve, CLSM 02297-penta. 6) VENEZIA, Caorle, Brussa, $0 \mathrm{~m}$, on hare dung in d.c., A. Bizzi, 27.10.07, 107.2-Ca Corniani, CLSM 02297-esa. 7) PISA, Orciatico, 50 m, on sheep dung in d.c., F. Doveri, 13.4.09, 285.3-Lajatico, CLSM 02297-epta. 8) BELLUNO, Sappada, 1200 m, on badger dung in d.c., L. Levorato, 16.8.10, 031.4-Prato Carnico, CLSM 02297octo.

TOTAL 8: wild rabbit 3; hare 2; badger 1; roe deer 1; sheep 1. 
Chaetomium elatum Kunze: Fr, Syst. Mycol. 3: 2541829.

MATERIAL: 1) LECCE, Vanze, $10 \mathrm{~m}$, about ten scattered, superficial specimens, on horse dung, V. Sciurti \& F. Doveri, 24.11.01, 513.4-S. Foca, CLSM 002.02. 2) PISA, Orciatico, 50 m, on sheep dung in d.c., F. Doveri, 13.4.09, 285.3-Lajatico, CLSM 002.02-bis. 3) PISA, Monteverdi Marittimo, Allumiere farm, 200 m, on rabbit dung in d.c., F. Doveri, 25.3.11, 306.4-Mounteverdi Marittimo, CLSM 002.02-ter. 4) VICENZA, Sarego, Mount Roccolo, 270 m, on cricket dung in d.c., A. Bizzi, 5.5.12, 146.4-Lonigo, CLSM 002.02-quater.

TOTAL 4: cricket 1; horse 1; rabbit 1; sheep 1 .

Chaetomium funicola Cooke, Grevillea 1: 1761873.

MATERIAL: 1) VICENZA, Posina, ?, about fifty gregarious specimens on roe deer dung in d.c., A. Bizzi, 25.5.02, 081.2-Castana, CLSM 021.02. 2) FERRARA, Mesola-Lido di Volano, 0 $\mathrm{m}$, on horse dung in d.c., A. Bizzi, 9.11.06, 187.1-Mesola, CLSM 021.02 bis. 3) LIVORNO, Suvereto, $50 \mathrm{~m}$, on horse dung in d.c., F. Doveri, 18.3.09, 305.2-San Vincenzo Sud, CLSM 021.02 ter. 4) VICENZA, Tonezza del Cimone, Fiorentini upland, $1450 \mathrm{~m}$, on hare dung in d.c., A. Bizzi, 13.7.08, 082.3-Arsiero, CLSM 021.02-quater.

TOTAL 4: horse 2; hare 1; roe deer 1.

Chaetomium fusisporum G. Sm., Trans. Brit. Mycol. Soc. 44: 461961.

MATERIAL: 1) NUORO, Lanusei, on sheep dung in d.c., A. Bizzi, 10.11.99, 531.2-Lanusei, CLSM 06598 ter. 2) FOGGIA, San Marco in Lámis, 700 m, on hare dung in d.c., L. Levorato, 28.5.12, 396.1-San Marco in Lámis, CLSM 06598-octo.

TOTAL 2: hare 1; sheep 1.

Chaetomium gangligerum L.M. Ames, Mycologia 41: 6401949.

MATERIAL: 1) MESSINA, Novara di Sicilia (Loc. Serra Mandrazze), about thirty gregarious, superficial specimens on cervine dung, G. Robich, 11.11.98, 613.4-Francavilla di Sicilia, CLSM 06598. 2) CATANIA, Etna Park, 1800 m, on sheep dung in d.c., A. Bizzi, 9.11.98, 612.2-Randazzo, CLSM 06598 bis. 3) COSENZA, Rossano (Cozzo del Pesco), $900 \mathrm{~m}$, on goat dung in d.c., C. Lavorato, 22.9.00, 552.1-Corigliano Calabro, CLSM 06598 quater. 4) COSENZA, loc. Pettinascura-S. Giovanni in Fiore, $1600 \mathrm{~m}$, on roe deer dung in d.c., C. Lavorato, 26.2.01, 560.1-Monte Volpintesta, CLSM 06598 penta. 5) REGGIO CALABRIA, Tazza, $800 \mathrm{~m}$, on cattle dung in d.c., F. Doveri, 17.10.03, 602.3-Motta San Giovanni, CLSM 06598 esa.

TOTAL 5: cattle 1; deer 1; goat 1; roe deer 1; sheep 1.

Chaetomium globosum Kunze: Fr, Syst. Mycol. 3: 2551829.

MATERIAL: 1) REGGIO CALABRIA, Tazza (Aspromonte), $800 \mathrm{~m}$, about fifty gregarious, superficial specimens, on sheep dung in d.c., F. Doveri, 17.10.03, 602.3-Motta San Giovanni, CLSM 001.03 ter. 2) BELLUNO, Campon-Farra d'Alpago, $1000 \mathrm{~m}$, on horse dung in d.c., A. Bizzi, 5.7.03, 064.4-Farra d'Alpago, CLSM 001.03 quater. 3) VICENZA, Enego, 850 m, on cattle dung, F. Doveri, 5.6.04, 083.4-Arsié, CLSM 001.03 penta. 4) VICENZA, Enego-Marcesina, 1300 m, on hare dung, F. Doveri, 5.6.04, 083.4-Arsié, CLSM 001.03 esa. 5) COSENZA, S. Sofia d'Epiro-Serra di Zoto, $600 \mathrm{~m}$, on hedgehog dung in d.c., C. Lavorato, 6.8.04, 552.4-S. Demetrio Corone, CLSM 001.03 epta. 6) COSENZA, S. Demetrio Corone, $800 \mathrm{~m}$, on sparrow dung in d.c., C. Lavorato, 23.11.03, 552.4-S. Demetrio Corone, CLSM 001.03 octo. 7) VICENZA, LongareMount Brosimo, 100 m, on dormouse dung in d.c., A. Bizzi, 6.6.06, 125.2-Longare, CLSM 001.3 
ena. 8) FERRARA, Mesola-Lido di Volano, 0 m, on horse dung in d.c., A Bizzi, 9.11.06, 187.1Mesola, CLSM 001.03 deca. 9) REGGIO EMILIA, Pratizzano Pass, $1200 \mathrm{~m}$, on hare dung in d.c., L. Levorato, 23.5.08, 217.2-Ramiseto, CLSM 001.03-XI. 10) REGGIO EMILIA, Pratizzano Pass, $1200 \mathrm{~m}$, on badger dung in d.c., G. Robich, 23.5.08, 217.2-Ramiseto, CLSM 001.03-XII. 11) VICENZA, Longare, Mount Brosimo, $100 \mathrm{~m}$, on dormouse dung in d.c., A. Bizzi, 6.6.11, 125.2Longare CLSM 001.03-XIII. 12) PISA, Orciatico, 50 m, on sheep dung in d.c., F. Doveri, 13.4.09, 285.3-Lajatico, CLSM 001.03-XIV. 13) GROSSETO, Gavorrano station, $75 \mathrm{~m}$, on cattle dung in d.c., F. Doveri, 25.3.11, 318.1-Gavorrano, CLSM 001.03-XV. 14) GROSSETO, Massa Marittima, $300 \mathrm{~m}$, on sheep dung in d.c., F. Doveri, 25.3.11, 306.2-Massa Marittima, CLSM 001.03-XVI. 15) LIVORNO, Elba island, $\mathrm{m}$ ?, on wild rabbit dung in d.c., L. Levorato, 9.4.11, ?, CLSM 001.03XVII. 16) LIVORNO, Capraia island, $0 \mathrm{~m}$, on hedgehog dung in d.c., L. Levorato, 11.4.11, 317.4Populonia, CLSM 001.03-XVIII.

TOTAL 16: sheep 3; cattle 2; dormouse 2; hare 2; hedgehog 2; horse 2; badger 1; sparrow 1; wild rabbit 1.

Chaetomium homopilatum Omvik, Mycologia 47: 7491955.

MATERIAL: VICENZA, Ponna, $600 \mathrm{~m}$, more than fifty gregarious, superficial specimens, on roe deer dung in d.c. or on blotting paper around its base, A. Bizzi, 18.07.04, 102.1-Recoaro Terme, CLSM 002.05.

TOTAL 1: roe deer.

Chaetomium medusarum J.A. Mey. \& Lanneau, Bull. Soc. Mycol. Fr. 83: 3181967.

MATERIAL: COSENZA, S. Demetrio Corone (Contrada Poggio), $600 \mathrm{~m}$, dozens of gregarious, superficial specimens, on sparrow dung in d.c., C. Lavorato, 26.5.98, 552.4-S. Demetrio Corone, CLSM 05898.

TOTAL 1: sparrow.

Chaetomium mollicellum L.M. Ames, Monogr. Chaetom.: 301963.

MATERIAL: 1) COSENZA, Rossano, $700 \mathrm{~m}$, dozens of gregarious, superficial specimens on wild pig dung in d.c., C. Lavorato, 22.12.98, 552.1-Corigliano Calabro, CLSM 00799. 2) COSENZA, S. Benedetto Acri, $700 \mathrm{~m}$, on hedgehog dung in d.c., C. Lavorato, 7.8.98, 552.4-S. Demetrio Corone, CLSM 00799 bis. 3) COSENZA, S. Demetrio Corone, $800 \mathrm{~m}$, on rat dung in d.c., C. Lavorato, 29.2.00, 552.4-S. Demetrio Corone, CLSM 00799 ter. 4) COSENZA, S. Demetrio Corone, $800 \mathrm{~m}$, on lizard dung in d.c., C. Lavorato, 19.8.01, 552.4-S. Demetrio Corone, CLSM 00799 quater. 5) COSENZA, Corigliano Calabro-loc. Tenimento, $250 \mathrm{~m}$, on lizard dung in d.c., C. Lavorato, 16.11.01, 552.1-Corigliano Calabro, CLSM 00799 penta. 6) COSENZA, Acri, $1050 \mathrm{~m}$, on beech-marten dung in d.c., C. Lavorato, 30.4.02, 552.3-Acri, CLSM 00799 esa. 7) COSENZA, Acri, $1000 \mathrm{~m}$, on wild pig dung in d.c., C. Lavorato, 10.5.02, 552.3-Acri, CLSM 00799 epta. 8) VICENZA, Cesuna, Roana, $m$ ?, on fox dung in d.c., G. Robich, 6.7.08, 082.2Asiago, CLSM 00799-octo.

TOTAL 8: lizard 2; wild pig 2; beech-marten 1; fox 1; hedgehog 1; rat 1.

Chaetomium murorum Corda, Icon. Fung. 1: 241837.

MATERIAL: 1) VICENZA, Villaga-Mount Tondo, $250 \mathrm{~m}$, about ten gregarious, superficial specimens, on hedgehog dung in d.c., A. Bizzi, 19.8.07, 125.2-Longare, CLSM 010.08. 2) ASTI, Agliano Terme, $250 \mathrm{~m}$, on bat dung in d.c., M. Filippa, 20.6.05, 193.1-Canelli, CLSM 010.08 bis. 3) VICENZA, Lumignano, Mount Brosimo, $100 \mathrm{~m}$, on dormouse dung in d.c., A. Bizzi, 24.11.08, 125.2-Longare, CLSM 010.08 ter 4) LIVORNO, Montioni, $0 \mathrm{~m}$, on rabbit dung in 
d.c., F. Doveri, 18.3.09, 306.3-Suvereto, CLSM 010.08 quater. 5) LIVORNO, Bibbona, Fattoria di Faltona, 50 m, on sheep dung in d.c., F. Doveri, 18.3.09, 295.3-Sassa, CLSM 010.08 penta. 6) PISA, Orciatico, $50 \mathrm{~m}$, on sheep dung in d.c., F. Doveri, 13.4.09, 285.3-Lajatico, CLSM 010.08 esa. 7) PISA, Volterra, Villamagna, Vallicella farm, $200 \mathrm{~m}$, on cattle dung in d.c., F. Doveri, 13.4.09, 285.3-Lajatico, CLSM 010.08 epta. 8) PISA, Volterra, Villamagna, Vallicella farm, 200 $\mathrm{m}$, on sheep dung in d.c., CLSM 010.08 octa. 9) VICENZA, Tonezza del Cimone, Fiorentini upland, $1450 \mathrm{~m}$, on hare dung in d.c., A. Bizzi, 13.7.08, 082.3-Arsiero, CLSM 010.08 ena. 10) VICENZA, Solagna, Campeggia, $980 \mathrm{~m}$, on mouflon dung in d.c., A. Bizzi, 2.6.09, 083.3-Mount Grappa, CLSM 010.08 deca. 11) VICENZA, Longare, Lumignano, Mount Brosimo, $350 \mathrm{~m}$, on snail dung in d.c., A. Bizzi, 11.6.09, 125.2-Longare, CLSM 010.08-XI. 12) VICENZA, Longare, Lumignano, Mount Brosimo, $350 \mathrm{~m}$, on dormouse dung in d.c., 22.6.09, CLSM 010.08-XII. 13) NOVARA, Druogno, Santa Maria, $850 \mathrm{~m}$, on sheep dung in d.c., F. Doveri, 15.5.10, 116.1Bellinzago, CLSM 010.08-XIII. 14) BELLUNO, Sappada, $1200 \mathrm{~m}$, on badger dung in d.c., L. Levorato, 16.8.10, 031.4-Prato Carnico, CLSM 010.08-XIV. 15) GROSSETO, Ribolla, Collecchia, $50 \mathrm{~m}$, on sheep dung in d.c., F. Doveri, 25.3.11, 319.4-Ribolla, CLSM 010.08-XV. 16) PISA, Mountrotondo Marittimo, Capanni, $100 \mathrm{~m}$, on sheep dung in d.c., F. Doveri, 26.4.11, 306.4Monteverdi Marittimo, CLSM 010.08-XVI. 17) PISA, Monteverdi Marittimo, Boraciferous lake, $250 \mathrm{~m}$, on cattle dung in d.c., F. Doveri, 25.3.11, 306.4-Monteverdi Marittimo, CLSM 010.08XVII. 18) PISA, Monteverdi Marittimo, Boraciferous lake, $250 \mathrm{~m}$, on cattle dung in d.c., F. Doveri, 25.3.11, 306.4-Monteverdi Marittimo, CLSM 010.08-XVIII. 19) GROSSETO, Stazione di Gavorrano, 75 m, on cattle dung in d.c., F. Doveri, 25.3.11, 318.1-Gavorrano, CLSM 010.08-XIX. 20) PISA, Monteverdi Marittimo, Boraciferous lake, $250 \mathrm{~m}$, on cattle dung in d.c., F. Doveri, 25.3.11, 306.4-Monteverdi Marittimo, CLSM 010.08-XX. 21) GROSSETO, Massa Marittima, 300 $\mathrm{m}$, on sheep dung in d.c., F. Doveri, 25.3.11, 306.2-Massa Marittima, CLSM 010.08-XXI. 22) PISA, Monteverdi Marittimo, Allumiere farm, $200 \mathrm{~m}$, on rabbit dung in d.c., F. Doveri, 25.3.11, 306.4-Monteverdi Marittimo, CLSM 010.08-XXII. 23) TRENTO, Vervò, Predaia refuge, 1300 m, on hare dung in d.c., A. Bizzi, 3.10.10, 043.4-Cles, CLSM 010.08-XXIII. 24) LIVORNO, Elba island, $\mathrm{m}$ ?, on sheep dung in d.c., L. Levorato, 9.4.11, ?, CLSM 010.08-XXIV. 25) LIVORNO, Capraia island, $0 \mathrm{~m}$, on hedgehog dung in d.c., L. Levorato, 11.4.11, 317.4-Populonia, CLSM 010.08-XXV. 26) VICENZA, Sarego, Mount Roccolo, 270 m, on cricket dung in d.c., A. Bizzi, 5.5.12, 146.4-Lonigo, CLSM 010.08-XXVI.

TOTAL 26: sheep 8; cattle 5; dormouse 2; hare 2; hedgehog 2; rabbit 2; badger 1; bat 1; cricket 1; moufloun 1; snail 1 .

Chaetomium oblatum Dreyfuss \& Arx in von Arx et al., Nova Hedwigia, beih. 84: 61986.

MATERIAL: COSENZA, Orsomarso, $1300 \mathrm{~m}$, about ten gregarious, superficial specimens, on blotting paper around the base of donkey dung in d.c., C. Lavorato, 17.5.98, 533.2-Mormanno, CLSM 05698.

TOTAL 1: donkey.

Chaetomium robustum L.M. Ames, Monogr. Chaetom.: 351963.

MATERIAL: 1) COSENZA, Corigliano Calabro-loc. Tenimento, $250 \mathrm{~m}$, dozens of gregarious, superficial specimens, on rabbit dung in d.c., C. Lavorato, 12.1.02, 552.1-Corigliano Calabro, CLSM 008.02. 2) COSENZA, S. Demetrio Corone-Contrada Calamia, $800 \mathrm{~m}$, on lizard dung in d.c., C. Lavorato, 19.10.01, 552.4-S. Demetrio Corone, CLSM 008.02 bis. 3) VICENZA, Mount Brosimo-Lumignano, $100 \mathrm{~m}$, on dormouse dung in d.c., A. Bizzi, 12.6.03, 125.2-Longare, CLSM 008.02 ter. 4) VICENZA, Recoaro, $1000 \mathrm{~m}$, on rabbit dung in d.c., A. Bizzi, 22.5.04, 102.2Valdagno, CLSM 008.02 quater. 5) VICENZA, Malga Melegnon-Lestebasse, $1300 \mathrm{~m}$, on roe deer dung in d.c., A. Bizzi, 3.9.04, 081.1-Caldonazzo, CLSM 008.02-penta. 6) VICENZA, LongareMount Brosimo, 100 m, on dormouse dung in d.c., A. Bizzi, 6.6.06, 125.2-Longare, CLSM 008.02- 
esa. 7) VICENZA, Villaga, Mount Tondo, $250 \mathrm{~m}$, on badger dung, A. Bizzi, 11.8.07, 125.2Longare, CLSM 008.02-epta. 8) REGGIO EMILIA, Pratizzano Pass, $1200 \mathrm{~m}$, on badger dung in d.c., G. Robich, 23.5.08, 217.2-Ramiseto, CLSM 008.02-octa. 9) VICENZA, Longare, Lumignano, Mount Brosimo, $350 \mathrm{~m}$, on snail dung in d.c., A. Bizzi, 11.6.09, 125.2-Longare, CLSM 008.02ena. 10) VICENZA, Longare-Mount Brosimo, $100 \mathrm{~m}$, on dormouse dung in d.c., A. Bizzi, 2.6.10, 125.2-Longare, CLSM 008.02-deca. 11) VENEZIA, Ca Savio, $0 \mathrm{~m}$, on hare dung in d.c., L. Levorato, 23.3.10, 128.3-Venezia, CLSM 008.02-XI. 12) PISA, Montopoli Val d'Arno, $150 \mathrm{~m}$, on hare dung in d.c., F. Doveri, 25.6.11, 274.3-Montopoli in Val d'Arno, CLSM 008.02-XIII. 13) LIVORNO, Pianosa island, $0 \mathrm{~m}$, on wild rabbit dung in d.c., L. Levorato, 8.4.11, 341.4-Isola di Pianosa, CLSM 008.02-XIV. 14) GROSSETO, Giglio island, loc. Castello, $400 \mathrm{~m}$, on wild rabbit dung in d.c., S. Rum, 352.4-Giglio, CLSM 008.02-XV. 15) LIVORNO, Elba island, ?, on sheep dung in d.c., L. Levorato, 9.4.11, ?, CLSM 008.02-XVI. 16) LIVORNO, Elba island, ?, on wild rabbit dung in d.c., L. Levorato, 9.4.11, ?, CLSM 008.02-XVII. 17) LIVORNO, Capraia island, 0 $\mathrm{m}$, on sheep dung in d.c., L. Levorato, 11.4.11, 317.4-Populonia, CLSM 008.02-XVIII. 18) PISA, La Sassa, 350 m, on roe deer dung in d.c., F. Doveri, 26.10.12, 295.3-Sassa, CLSM 008.02-XIX. 19) LIVORNO, Quercianella, $0 \mathrm{~m}$, on fallow deer dung in d.c., F. Doveri, 22.12.12, 284.3Rosignano marittimo, CLSM 008.02-XX. 20) LIVORNO, Capraia island, $0 \mathrm{~m}$, on hedgehog dung in d.c., L. Levorato, 11.4.11, 317.4-Populonia, CLSM 008.02-XXI. 21) VENEZIA, Eraclea Mare, 0 $\mathrm{m}$, on hare dung in d.c., L. Levorato, 6.11.10, 129.4-Eraclea, CLSM 008.02-XXII.

TOTAL 21: dormouse 3; hare 3; wild rabbit 3; badger 2; rabbit 2; roe deer 2; sheep 2; fallow deer 1 ; hedgehog 1; lizard 1; snail 1.

Chaetomium semen-citrulli Sergeeva, Not. Syst. Sect. Crypt. Inst. Bot. Acad. Sci. U.R.S.S. 11: 1131956.

MATERIAL: 1) COSENZA, S. Demetrio Corone (Contrada Poggio), $600 \mathrm{~m}$, dozens of gregarious, superficial specimens on sparrow dung in d.c., C. Lavorato, 26.5.98, 552.4-S. Demetrio Corone, CLSM 05798. 2) COSENZA, S. Demetrio Corone (Contrada Calamia), $800 \mathrm{~m}$, on weasel dung in d.c., C. Lavorato, 6.9..01, 552.4-S. Demetrio Corone, CLSM 05798 bis. 3) Cosenza, S. Sofia d'Epiro-loc. Quercia Rotonda, $250 \mathrm{~m}$, on porcupine dung in d.c., C. Lavorato, 12.7.05, 552.4S. Demetrio Corone, CLSM 05798 ter.

TOTAL 3: porcupine 1; sparrow 1; weasel 1.

Chaetomium spinosum Chivers, Proc. Am. Acad. Arts Sciences 48: 861912.

MATERIAL: VERONA, Bosco Chiesanuova-loc. Podesteria (Malga), $1600 \mathrm{~m}$, about ten isolated, superficial specimens on cattle dung in d.c., A. Bizzi, 1.6.06, 102.3-Bosco Chiesanuova, CLSM 005.07.

TOTAL 1: cattle.

Chaetomium subaffine Sergeeva, Not. Syst. Sect. Crypt. Inst. Bot. Acad. Sci. U.S.S.R. 14: 148 1961.

MATERIAL: 1) VERONA, Bosco Chiesanuova-loc. Podesteria (Malga), $1600 \mathrm{~m}$, three isolated, superficial specimens on cattle dung in d.c., A. Bizzi, 1.6.06, 102.3-Bosco Chiesanuova, CLSM 006.07. 2) VERONA, Bosco Chiesanuova-loc. Mount Tomba, $1750 \mathrm{~m}$, about ten specimens on cattle dung in d.c., A. Bizzi, 1.6.06, 102.3-Bosco Chiesanuova, CLSM 006.07 bis. 3) PISA, Volterra, Villamagna-Podere Vallicella, $200 \mathrm{~m}$, on pig dung in d.c., F. Doveri, 13.4.09, 285.3Lajatico, CLSM 006.07-ter. 4) BELLUNO, Sappada, 1200 m, on badger dung in d.c., L. Levorato, 16.8.10, 031.4-Prato Carnico, CLSM 006.07-quater.

TOTAL 4: cattle 2; badger 1; pig 1 . 


\section{Chaetomium succineum}

MATERIAL: LIVORNO, Elba island, $m$ ?, one superficial specimen on sheep dung in d.c., L. Levorato, 8.4.11, ?, CLSM 003.12.

TOTAL 1: sheep.

Chaetomium trigonosporum (Marchal) Chivers, Mem. Torrey Bot. Club 14: 1661915.

MATERIAL: 1) COSENZA, S. Demetrio Corone (Contrada Calamia), $800 \mathrm{~m}$, about ten gregarious, superficial specimens, on polecat dung in d.c., C. Lavorato, 10.8.01, 552.4-S. Demetrio Corone, CLSM 016.01.

TOTAL 1: polecat.

Chaetomium variostiolatum A. Carter, Can. J. Bot. 61: 26031983.

MATERIAL: 1) VERONA, Bosco Chiesanuova-loc. Mount Tomba, $1750 \mathrm{~m}$, dozens of isolated or gregarious, superficial specimens on cattle dung in d.c., A. Bizzi, 1.6.06, 102.3-Bosco Chiesanuova, CLSM 008.07. 2) VICENZA, Schio, S. Caterina di Tretto, $800 \mathrm{~m}$, on cattle dung in d.c., A. Bizzi, 1.9.07, 103.4-Malo, CLSM 008.07 bis. 3) REGGIO EMILIA, Pratizzano Pass, 1200 $\mathrm{m}$, on hare dung in d.c., L. Levorato, 23.5.08, 217.2-Ramiseto, CLSM 008.07 ter.

TOTAL 3: cattle 2; hare 1.

\section{Updated key to coprophilous Chaetomium from Italy}

[partly based on von Arx et al. 1986 and Doveri 2004, 2008; between square brackets, year of publication and linked colour photos(c.p.) or freehand drawings (f.d.)]

1) Ascospores triangular in frontal view, 8.5-9.5 × 4.5-5.5 ×4-4.5 $\mu \mathrm{m}(9-12 \times 5-7 \times 4-5$, von Arx et al.). Peridium cephalothecoid C. trigonosporum [2004 f.d., 2008 c.p.] 1*) Ascospores different in shape ...2

2) Ascospores oblate, subglobose in frontal view, ellipsoidal with rounded ends in side view, $7.5-8 \times 7.5 \times 4.5-5 \mu \mathrm{m}$ (6-7.5 $\times 4-5$, von Arx et al.), with one, indistinct germ pore. Peridium of a textura angularis. Terminal hairs $2-2.5 \mu \mathrm{m}$ diam., spirally coiled and circinate at the apex C. oblatum [2004 f.d., 2008 f.d.]

2*) Ascospores different in shape, with one, usually distinct germ pore 3

3) Asci narrowly cylindric. Ascospores uniseriate, broadly ovoidal, bilaterally flattened. Peridium of a textura angularis. Terminal hairs spirally coiled 21 3*) Asci clavate. Ascospores biseriate to conglobate, variously shaped. Peridium variously textured. Terminal hairs of several types

4) Ascospores with an apical germ pore

4*) Ascospores with an eccentric (subapical to clearly lateral) germ pore ......................................................18

5) Ascomata with a dark collar around the ostiole. Peridium of a textura angularis. Ascospores dextrinoid in the early stages, pale grey at maturity, limoniform (sometimes subglobose), biapiculate, bilaterally flattened ..........................6

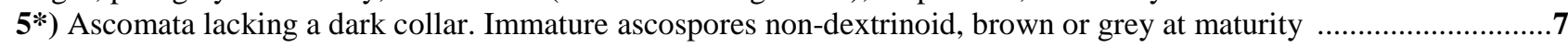
6) Ascomata usually ovoidal or ellipsoidal. Peridium all of a textura angularis. Terminal hairs with spirally coiled, often branched ends (branches in turn spirally coiled). Ascospores 7-7.5 × 5.5-6.5 $\mu \mathrm{m}(5.5-7.5 \times 5-6.5$, von Arx et al.) ......... C. bostrychodes [2004 f.d., 2008 c.p.]

6*) Ascomata usually ampulliform. Upper peridial portion a textura prismatica of elongated cells in vertical rows. Terminal hairs spirally coiled, but rarely branched. Ascospores 6-6.5 × 5-6 $\mu \mathrm{m}(5.5-7.5 \times 5-6.5$, von Arx et al.) ..C. robustum [2004 f.d., c.p.; 2008 c.p.] 7) Ascomata ampulliform with an elongated, cylindric-conical neck. Peridium of a textura angularis. Terminal hairs seta-like, unbranched. Ascospores limoniform, brown, 7-9 × 5.5-7 × 4.8-5.2 $\mu \mathrm{m}(6.5-8.5 \times 6-7.5 \times 5-6$, von Arx et al.) …............................................................................................................ homopilatum [2008 c.p.] $\left.7^{*}\right)$ Ascomata globose to broadly ellipsoidal or ovoidal, lacking an elongated neck. Peridium variously textured ...........8 8) Ascospores 13-14.5 × 11-12.5 × 8-10 $\mu \mathrm{m}(12-16 \times 9-11 \times 7-10$, von Arx et al.), brown, watermelon seed-shaped, broadly ovoidal in frontal view, broadly ellipsoidal in side view. Peridium of a textura angularis. Terminal hairs spirally coiled C. semen-citrulli [2004 f.d., 2008 c.p.]

8*) Ascospores smaller and/or different in shape

9) Ascospores brown, limoniform, biapiculate, bilaterally flattened. Terminal hairs not seta-like ................................10 9*) Ascospores brown or grey, ellipsoid-fusiform or ovoidal, rarely limoniform, then mixed with ovoidal ascospores ..... 
10) Colonies lacking greenish-yellow exudates, at most with hairs shading from brown into these colours. Terminal hairs unbranched, wavy to flexuous. Peridium of a textura intricata or epidermoidea at intervals. Ascospores $10-12 \times$ 7.5-9 × 7-8 $\mu \mathrm{m}(11-15 \times 8-11 \times 7-8.5$, von Arx et al. $)$ C. subaffine [2008 c.p.]

10*) Colonies often with greenish-yellow exudates. Terminal hairs differently shaped

11) Peridium usually of a textura epidermoidea. Terminal hairs flexuous and dichotomously branched. Ascospores $11-$ $11.5 \times 7.5-8.5 \times 6.5-7 \mu \mathrm{m}(11-14 \times 8-11 \times 7-9$, von Arx et al. $)$ C. elatum $[2004$ f.d., 2008 c.p.] 11*) Peridium of a textura intricata. Terminal hairs flexuous or more often spirally coiled, unbranched. Ascospores 810 x 6.5-9.5 × 5.5-7.5 $\mu \mathrm{m}(9-12 \times 8-10 \times 6-8$, von Arx et al. $)$ C. globosum [2004 f.d., 2008 c.p.]

12) Ascospores ellipsoid-fusiform, with attenuated, sometimes apiculate ends

12*) Ascospores different in shape

13) Ascospores greyish blue, $8.5-10.5 \times 4.8-5.2 \mu \mathrm{m}(7-10 \times 4.5-6$, von Arx et al.). Peridium of a textura angularis. Terminal hairs of two kinds: shorter and arcuate, or longer and wavy .... C. carinthiacum [2008 c.p.] 13*) Ascospores larger, greyish brown. Terminal hairs different in shape

14) Peridium of a textura cephalothecoidea. Terminal hairs straight, open circinate at the apex. Ascospores sometimes slightly inequilateral, $15.5-17.5 \times 9-10.5 \mu \mathrm{m}$ C. ancistrocladum [2011 c.p.]

14*) Peridium and terminal hairs different .15

15) Peridium of a textura angularis. Terminal hairs undulate to loosely spirally coiled. Ascospores slightly inequilateral, $13-13.5 \times 7.5-8 \mu \mathrm{m}(12-17 \times 6-8.5$, von Arx et al. $)$

C. succineum

15*) Peridium of a textura angularis to epidermoidea, sometimes cephalothecoidea around the hair bases. Terminal hairs flexuous at the apex and often open circinate. Ascospores usually equilateral, $13-16 \times 7.5-9 \mu \mathrm{m}(13-17 \times 7-9$ von Arx et al.)

C. murorum [2011 c.p.]

16) Ascomata ostiolate. Peridium with an outer layer of textura intricata. Terminal hairs of two kinds: seta-like and unbranched, or dichotomously branched. Ascospores limoniform or ovoidal, dark grey, 5.5-6 $\times 3.8-4.2 \times 3-3.5 \mu \mathrm{m}$ (brown, 6-7.5 × 4-5.5, von Arx et al.)

C. funicola [2004 f.d., 2008 c.p.]

16*) Ascomata non-ostiolate or ostiolate at late maturity. Terminal hairs seta-like, usually unbranched. Ascospores ovoidal.

17) Ascomata ostiolate at late maturity. Peridium of a textura angularis, cephalothecoidea at intervals. Ascospores 6-7 $\times$ 3-4 $\mu \mathrm{m}(5.5-7.5 \times 4-5$, von Arx et al.), ovoidal to narrowly ovoidal, rarely flattened at one side, non-apiculate, pale greyish.

C. spinosum [2008 c.p.] $17 *)$ Ascomata non-ostiolate or ostiolate at late maturity only. Peridium of a textura intricata to epidermoidea. Ascospores 5.5-6.5 × 4-4.5 × 3-3.5 $\mu \mathrm{m}$ (5.5-6.5 × 3.5-4.5 × 3-3.5, von Arx et al.), broadly ovoidal, uni- or bilaterally flattened, apiculate, pale brown to greyish.

C. variostiolatum [2008 c.p.] 18) Ascospores with an oblique to slightly eccentric germ pore (central in our Italian collection), ellipsoid-fusiform, greyish-blue, $8.5-10.5 \times 4.8-5.2 \mu \mathrm{m}(7-10 \times 4.5-6$, von Arx et al.). Peridium of a textura angularis. Terminal hairs of two kinds: shorter and arcuate, or longer and wavy C. carinthiacum $[2008$ c.p]

18*) Ascospores with an eccentric to lateral germ pore. Terminal hairs different ...............................................19 19) Peridium of a textura angularis. Terminal hairs of two kinds: pale, strongly ramified, thin-walled; or darker, less ramified, thicker. Ascospores ellipsoid-narrowly limoniform $(Q=1.70), 9.5-11.5 \times 5.5-6.5 \mu \mathrm{m}(8-12 \times 5-7$, von Arx et al.) ................................................................................................. C. cuniculorum [2004 f.d., 2008 c.p.] 19*) Peridium with a superficial layer of textura intricata. Terminal hairs with long spirally coiled ends. Average Q larger

20) Ascospores ellipsoid-subfusiform, usually symmetrical, with rounded, often subumbonate ends, $14-17 \times 8-9.5 \mu \mathrm{m}$ $(12-15 \times 7.5-9$, von Arx et al.) .................................................................................. C. gangligerum [2008 c.p.] 20*) Ascospores fusiform, often inequilateral, with fairly pointed ends, 16-18.5 × 6-7 $\mu \mathrm{m}(14-17 \times 7-8$, von Arx et al.)

21) Ascospores $11-12 \times 8-9 \mu \mathrm{m}(8-11 \times 7-8$, von Arx et al.) Spirally coiled hairs unbranched

C. fusisporum [2004 f.d., 2008 c.p.] C. crispatum $[2004$ f.d., 2008 c.p.]

21*) Ascospores smaller

22) Terminal hairs 5-8 $\mu \mathrm{m}$ diam., with spirally coiled branches. Ascospores $7-7.5 \times 5.5-6.5 \mu \mathrm{m}(7-8.5 \times 6-7$, von Arx et al.) C. medusarum [2004 f.d., 2008 c.p.]

22*) Terminal hairs 4-6.5 $\mu \mathrm{m}$ diam., unbranched .23

23) Ascospores $9-9.5 \times 7.5-8 \mu \mathrm{m}(8-9.5 \times 7-8$, von Arx et al.) C. mollicellum [2004 f.d., 2008 c.p.]

23*) Ascospores 6.5-7.5 ×6-6.5 $\mu \mathrm{m}(7-8.5 \times 6-7$, von Arx et al.) C. brasiliense

\section{Updated world-key to Chaetomium spp. after von Arx et al. (1986) and Doveri (2008)}

(in bold type new species, new combinations, or taxa accepted in Chaetomium after von Arx et al.)

1) Ascospores triangular in frontal view, brown at maturity

2) Ascospores cylindrical, with attenuated ends, $9-11 \times 2.5-3 \mu \mathrm{m}$. Colonies often with red or orange exudates. 
Peridium of a textura intricata. Ascomatal hairs seta-like, dichotomously branched

2*) Ascospores not cylindrical, broader

3) Asci 2 - to 6-spored. Ascospores ellipsoidal, biumbonate, bilaterally flattened, $11-15 \times 7-9 \times 5-6 \mu$ m. Peridium of a textura angularis. Hairs flexuous or undulate, branched at the apex. Colonies with yellow exudates ..........C. tetrasporum 3*) Asci 8-spored

4) Ascospores oblate or hemispherical, 6-7.5 × 4-5 $\mu \mathrm{m}$, lacking a distinct germ pore. Peridium of a textura angularis. Ascomatal hairs spirally coiled C. oblatum

5) Asci clavate, obovate or fusiform. Ascospores biseriate or conglobate ...5

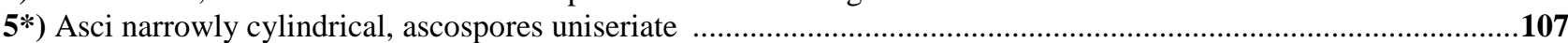

6) Ascospores spherical or subspherical, sometimes with two germ pores .........................................................

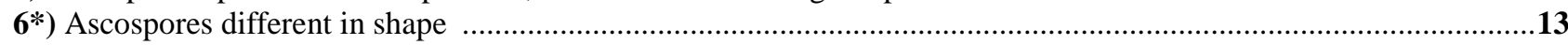

7) Ascospores with one (occasionally two) germ pore and usually a regular outline ................................................8

$7^{*}$ ) Ascospores with two (or more) germ pores and often an irregular outline .......................................................12

8) Ascospores $5.2-6.3 \times 5.5-7.8 \mu \mathrm{m}$. Terminal hairs irregularly or dichotomously branched

C. mesopotamicum Abdullah \& Zora 1993

8*) Ascospores larger

...9

9) Ascospores globose, 10-13 $\mu \mathrm{m}$ diam. Peridium of a textura angularis. Terminal hairs flexuous to undulate.

C. globosporum

9*) Ascospores nearly spherical to broadly ellipsoidal or ovoidal

.10

10) Ascomata subglobose. Peridium of a textura epidermoidea. Terminal hairs flexuous to undulate, $4-6 \mu \mathrm{m}$ diam. at the base. Ascospores 9-14 × 7-11 ×9-9.5 $\mu \mathrm{m}$, with a paler longitudinal band and a subapical to lateral germ pore $\ldots . . . .$.

C. tarraconense Stchigel et al. in Rodríguez et al. 2002

10*) Ascomata obovoidal. Peridium with a different textura. Ascospores without a longitudinal band

11) Peridium of a textura angularis or intricata. Terminal hairs flexuous, undulate or hypha-like, 4-7.5 diam. near the base. Ascospores 10-12.3 $\times 9-11.3 \times 7-9 \mu \mathrm{m}$, with an apical or slightly subapical germ pore

C. interruptum Asgari \& Zare 2011

11*) Peridium of a textura intricata. Terminal hairs of two types: short, rigid and staight; or long, sinuous, branched, both types 3-5 $\mu \mathrm{m}$ diam. near the base. Ascospores 8-11 $\times 8-9.5 \mu \mathrm{m}$, with a subapical or sometimes lateral germ pore

C. umbratile Udagawa et al. 1997

12) Ascospores $12-16 \times 10-14 \times 8-12 \mu \mathrm{m}$, subspherical to ovoidal, often bilaterally flattened, with two germ pores. Ascomatal hairs flexuous, or undulate, or hyphoid. Peridium of a textura angularis. Colonies occasionally with pale green exudates. Homothallic

C. megalocarpum 12*) Ascospores 15-22 × 11-16 $\mu \mathrm{m}$, broadly ellipsoidal with rounded ends to ovoidal, pyriform or subglobose, with two or more germ pores. Hairs hyphoid. Peridium of a textura epidermoidea. Colonies often with yellow exudates. Heterothallic C. nozdrenkoae N.B.: C. grande Asgari \& Zare (2011) appears, in my opinion, very similar to, or even the same species as C. nozdrenkoae Sergeeva (see also Sergejeva 1961, von Arx et al. 1986). Only the asci are described as larger in C. grande, whereas the peridium of this species, described as a textura angularis or irregularis in the protologue, actually is a textura epidermoidea (see Asgari \& Zare, figs. 6i-j) like that of C. nozdrenkoae (von Arx et al. 1986).

13) (from 6) Ascospores with one germ pore (occasionally with a paler spot at the end opposite to the germ pore) ......14

13*) Ascospores at least partly with two germ pores or lacking distinct germ pores ..............................................93

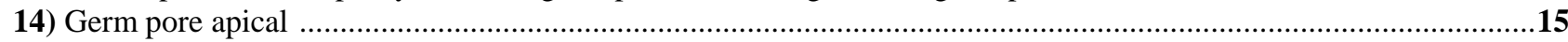

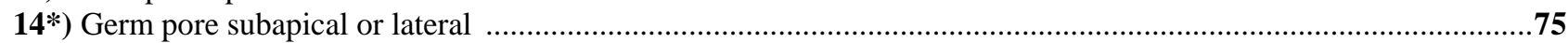

15) Ascospores rhomboid with umbonate ends, bilaterally flattened, $9-11 \times 6.5-8 \times 4.5-6 \mu$ m. Peridium pale, of a textura epidermoidea. Ascomatal hairs flexuous C. umbonatum

15*) Not with the above characters

. .16

16) Ascospores limoniform or irregular, usually biapiculate, bilaterally flattened, $9-14 \times 7-12 \mu$ m. Peridium with an

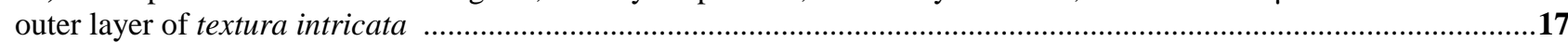

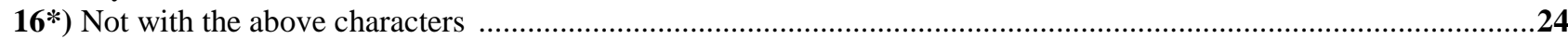

17) Ascomata with a very pale, yellowish peridium. Terminal hairs sparse, delicate, wavy, pale cream. Ascospores pink, red in mass, 7-10 × 7-7.5

C. cruentum

17*) Peridium pigmented. Terminal hairs dense. Ascospores brown at maturity ....................................................18

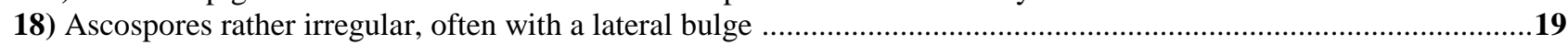

18*) Ascospores regular, limoniform, without a lateral bulge .....................................................................20

19) Colonies with yellow green exudates. Ascomata olivaceous in reflected light. Ascomatal hairs flexuous, undulate, or coiled. Ascospores with a lateral bulge, $9-11 \times 7-9 \times 6-7 \mu \mathrm{m}$........................................................... C. madrasense 19*) Colonies without exudates. Ascomata yellow in reflected light. Hairs hyphoid. Ascospores irregular, 8-12 x 5-7

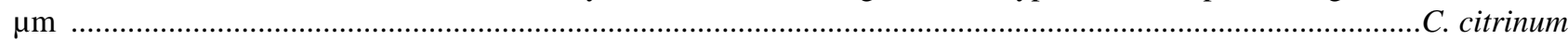

20) Homothallic. Terminal hairs flexuous, undulate or coiled. Phialoconidia absent .21

20*) Homothallic or heterothallic. Hairs branched or flexuous when unbranched. Phialoconidia present or absent .......22 21) Colonies usually with yellow-green or red exudates. Ascospores $9-12 \times 8-10 \times 6-8 \mu \mathrm{m}$

C. globosum (and its relatives) 
21*) Colonies without exudates. Ascospores 12-13.5 × 8-10 × 6-7.5 $\mu \mathrm{m}$

C. undulatulum Asgari \& Zare 2011

22) Heterothallic. Colonies lacking pigmented exudates. Terminal hairs unbranched, flexuous. Ascospores $11-15 \times 8-$

$11 \times 7-8.5 \mu \mathrm{m}$. Phialoconidia present

C. subaffine (and C. angustispirale)

22*) Terminal hairs dichotomously branched

23) Homothallic. Phialoconidia present. Colonies without exudates. Hairs usually branched at right angles. Ascospores

$9-11.5 \times 6.5-8.5 \times 5.5-6.5 \mu \mathrm{m}$

C. rectangulare Asgari \& Zare 2011

23*) Homothallic or eterothallic. Phialoconidia present, or absent in homothallic isolates. Colonies often with green or yellow exudates. Hairs irregularly branched. Ascospores $11-14 \times 8-11 \times 7-9 \mu \mathrm{m}$

C. elatum

24) (from 16) Ascospores 6-13 $\times 5-10 \mu \mathrm{m}$, limoniform, bilaterally flattened, umbonate or apiculate at the ends, brown (exceptionally pale grey) at maturity. Ascomata often ampulliform or pyriform and with a conical or cylindrical neck; peridium of a textura angularis (rarely and partly covered with a textura intricata) or occasionally epidermoidea ........25 24*) Not with the above characters

25) Ascomata with a long neck formed of joined setae . .35

25*) Ascomata without such a neck

26) Neck less than $250 \mu \mathrm{m}$ long. Ascospores $8-11 \times 7.5-9 \times 5-6 \mu \mathrm{m}$ with an apical germ pore

26*) Neck 300-2300 $\mu \mathrm{m}$ long

C. malayense

27) Ascospores limoniform, $9-12 \times 8-10 \times 7-8 \mu \mathrm{m}$, with an apical or slightly subapical germ pore ........ C. longicolleum

$27^{*}$ ) Ascospores limoniform to quadrangular in frontal view, 6-6.5 $\times 5-6 \times 3.5-4 \mu \mathrm{m}$, with an apical germ pore

C. cuyabenoense Decock \& Hennebert 1997

28) Ascomata with apical setae few in number. Ascospores $8-10 \times 7-8.5 \times 5-6 \mu \mathrm{m}$, biapiculate

C. seminudum

28*) Ascomata with dense apical setae or hairs

.29

29) Ascomata subglobose to ovoid, with a very large (up to $50 \mu \mathrm{m}$ diam.) ostiole. Terminal hairs arcuate or recurved at the apex, yellow to pale brown, unbranched. Peridium of a textura angularis to epidermoidea. Ascospores 10-13 $\times 7-$ $10 \times 6.5-7.5 \mu \mathrm{m}$, biapiculate C. macrostiolatum Stchigel et al. in Rodríguez et al. 2002

29*) Ascospores smaller. Terminal hairs darker ………......................................................................30

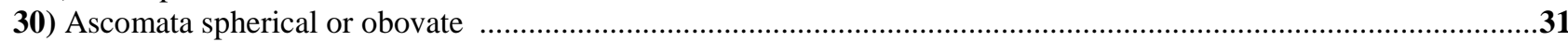

30*) Ascomata ovate to ampulliform . .32

31) Ascomata spherical. Peridium of a textura angularis. Ascomatal hairs flexuous or undulate, often branched.

Ascospores 7.5-9 × 6-7 × 4-5 $\mu \mathrm{m}$, brown, with an apical germ pore ...................................................... . sphaerale

31*) Ascomata obovate. Peridium of a textura angularis, partly covered with an outer layer of textura intricata.

Terminal hairs unbranched, constricted at the septa and articulated, loosely coiled at their tips. Ascospores 7-8.5 $\times 6-$

$7.2 \times 5-6 \mu \mathrm{m}$, pale grey, with an apical or slightly subapical germ pore

C. floriforme Gené \& Guarro 1996

32) Ascomata ovate or ampulliform. Hairs spirally coiled. Ascospores $6-7 \times 4.5-6 \times 3.5-4.5 \mu \mathrm{m}$

C. subspirale

32*) Ascomata ampulliform. Hairs not spirally coiled. Ascospores larger on average

. .33

33) Terminal hairs seta-like, straight, not branched. Ascospores 6.5-9.5 $\times 5.5-6.5 \times 3.5-4.5 \mu \mathrm{m}$, strongly umbonate.

Asci both clavate and cylindrical in the same ascoma .................. C. ampulliellum X.W. Wang in Wang \& Zheng 2005

33*) Terminal hairs often branched or circinate. Ascospores slightly umbonate. Asci always clavate

34) Hairs seta-like or flexuous, often branched or circinate at their apex. Ascospores 6.5-8.5 × 6-7.5 × 5-6 $\mu \mathrm{m}$

34*) Hairs seta-like, apically branched. Ascospores 8-9.5 × 4.5-6 $\mu \mathrm{m}$......................................................... ampullare

C. homopilatum

35) (from 24) Ascospores 5.5-10 $\times 5-8 \mu \mathrm{m}$, biapiculate or angular, bilaterally flattened, dextrinoid when young, pale

grey-olivaceous at maturity. Ascomata often with a darkened disc around the ostiole .....................................................36

35*) Ascospores not dextrinoid when young, usually brown at maturity. Ascomata lacking a darkened disc ................42

36) Ascomata small, spherical or oblate, pale. Ascomatal hairs partly short and verrucose, partly long, flexuous or undulate and smooth. Peridium of a textura angularis ..............................................................................................37 36*) Ascomata ovate, ampulliform or cylindrical, rarely spherical. Hairs usually spirally coiled at their apex and verrucose, often with some branches. Peridium of a textura angularis or cephalothecoidea

37) Ascospores 5.5-6.5 $\times 5-6 \times 3.5-5 \mu \mathrm{m}$, evenly pigmented

C. causiiforme

37*) Ascospores 7-9 $\times 6-7 \times 5-6 \mu \mathrm{m}$, darker near the ends

C. anguipilium

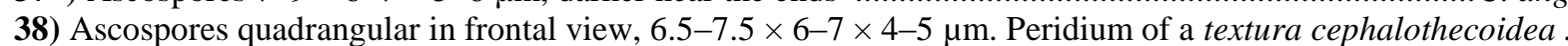

38*) Ascospores not quadrangular in frontal view

C. quadrangulatum

39) Ascomata ampulliform. Upper peridial portion a textura prismatica of dark, elongated cells in vertical rows.

Ascospores 5.5-7.5 ×5-6.5 ×4-5.5 $\mu \mathrm{m}$

C. robustum

39*) Upper peridial portion not formed of vertically elongated, dark cells . .40

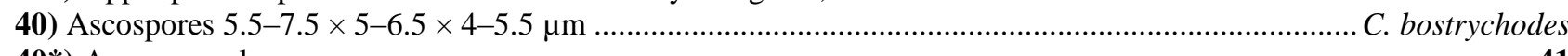

40*) Ascospores longer

41) Ascomata ovate or ampulliform. Textura cephalothecoidea. Ascospores $7-9.5 \times 6-7 \times 5-6 \mu \mathrm{m}$

C. convolutum

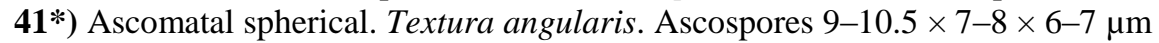

C. hexagonosporum

42) (from 35) Ascospores ovate, 5-8 $\times 4-6 \mu \mathrm{m}$, brown when mature, thick-walled. Peridium of a textura angularis often with an outer layer of textura intricata. Ascomatal hairs seta-like or/and dichotomously branched

42*) Not with the above characters 
43) Ascomatal hairs mainly seta-like, thick and stiff, with some lateral branches when old. Ascospores 6-8 $\times 4-5 \mu \mathrm{m}$, bilaterally not flattened

43*) Ascospores bilaterally flattened

44) Ascomatal hairs seta-like, unbranched. Ascomata occasionally not ostiolate. Ascospores 5.5-6.5 × 3.5-4.5 × 3-3.5 $\mu \mathrm{m}$

C. variostiolatum

44*) Ascomatal hairs at least partly dichotomously branched. Ascomata ostiolate

45) Ascomatal hairs partly seta-like, partly branched. Ascospores 6-7.5 × 4-5.5 × 3-4 $\mu \mathrm{m}$

C. funicola

45*) Nearly all hairs branched. Ascospores 5.5-7 × 4-5 × 3.5-4.5 $\mu \mathrm{m}$

C. indicum (and its relatives)

46) Peridium orange or pink. Ascomatal hairs much coiled. Ascospores ovate or pyriform, bilaterally flattened, brown,

$12-16 \times 9-11 \times 7-10 \mu \mathrm{m}$

C. semen-citrulli

46*) Not with the above characters

47) Ascomata nearly smooth, with a short cylindrical or conical neck. Peridium pale, reddish-brown. Ascospores irregular in shape, $12-13 \times 7-8 \mu \mathrm{m}$, greenish-brown, darkened around the germ pore

C. hamadae

47*) Not with the above characters

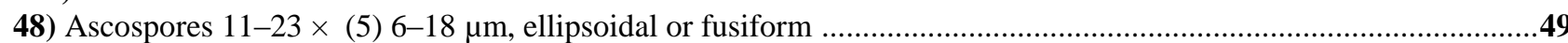

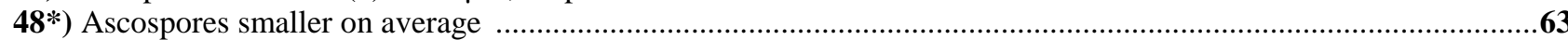

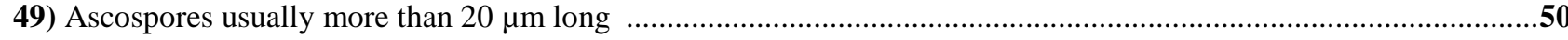

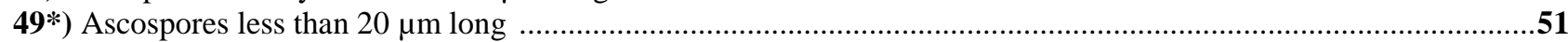

50) Ascomata brown. Peridium of a textura angularis. Ascomatal hairs brown, undulate, rarely branched, strongly constricted at their septa and articulate. Ascospores broadly fusiform, 20-22 × 16-18 $\mu \mathrm{m}$, with a protuberant, subapical (sometimes apical) germ pore, 2-2.5 $\mu \mathrm{m}$ wide ................................................. sinaiense Mustafa \& Ezz-Eldin 1989 50*) Ascomata pale ochraceous. Peridium of a textura epidermoidea or indistinctly angularis. Hairs pale and short or absent. Ascospores fusiform, 18-23 × 9-12 $\mu \mathrm{m}$, with an apical and smaller germ pore .............................. deceptivum 51) Ascospores 17-20 × 11-13 $\mu \mathrm{m}$, often ovoidal and occasionally with umbonate ends, laterally not flattened. Ascomata yellow or ochraceous. Peridium pale, with an outer layer of textura intricata. Ascomatal hairs hyphoid, sparse, flexuous

C. vitellinum

51*) Ascospores smaller .52

52) Colonies with yellow exudates. Ascomata non-ostiolate when young. Peridium translucent, a textura angularis of pale yellow or brown cells. Ascomata hairs hyphoid, often absent. Ascospores ellipsoidal, often irregular, $12-17 \times 7-$ $10 \mu \mathrm{m}$

C. irregulare

$\mathbf{5 2} *$ ) Not with the above characters

53) Peridium of a textura angularis. Ascomatal hairs seta-like, dark brown, unbranched. Ascospores fusiform, often inequilateral, 14-16 × 5-6 $\mu \mathrm{m}$, dark brown when mature, paler at the ends ............................................. dreyfussii

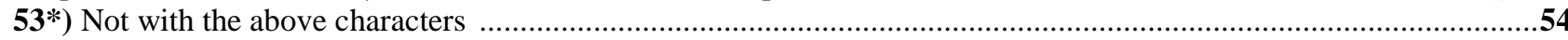

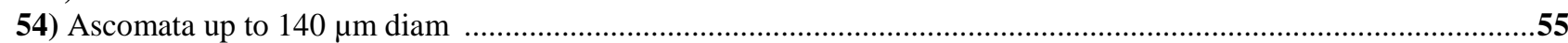

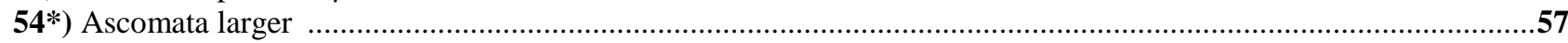

55) Terminal hairs tight spirally coiled all over the length. Ascospores fusiform, usually equilateral, with a truncated end around the germ pore, $11.5-13.5 \times 5.7-7 \mu \mathrm{m}$...................................................... truncatulum Asgari \& Zare 2011 $\mathbf{5 5} *)$ Terminal hairs not with such features. Ascospores larger on average, often inequilateral, without a truncate end $\mathbf{. 5 6}$ 56) Ascomatal hairs flexuous, undulate or spirally coiled, yellow green or olivaceous in reflected light. Ascospores ellipsoidal-fusiform, $13-16 \times 7-8 \mu \mathrm{m}$......................................................................................... subspirilliferum 56*) Ascomatal hairs hyphoid, straight or flexuous, dark olivaceous grey in reflected light. Ascospores fusiform, 13-15 $\times 6.5-8 \mu \mathrm{m}$ C. hispanicum 57) Anamorph Histoplasma-like. Ascomatal hairs straight to coiled. Ascospores ellipsoidal to narrowly ellipsoidal, $11.2-16 \times 7.2-9.6 \mu \mathrm{m}$ .. histoplasmoides Carris \& Glawe 1987

57*) Anamorph different or lacking

58) Anamorph Botryotrichum. Ascomatal hairs flexuous or apically circinate. Ascospores ellipsoidal with attenuated ends, $13-17 \times 8-10 \mu \mathrm{m}$ C. piluliferum

58*) Botryose aleurioconidia absent or rare 59

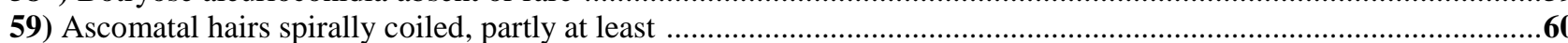

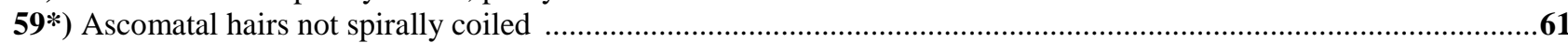

60) Terminal hairs tight spirally coiled all over the length. Ascospores fusiform, usually equilateral, with a truncated end around the germ pore, $11.5-13.5 \times 5.7-7 \mu \mathrm{m}$................................................... truncatulum Asgari \& Zare 2011 60*) Terminal hairs rather delicate, flexuous to spirally coiled. Ascospores ellipsoidal-fusiform, often inequilateral, without a truncated end, $12-17 \times 6-8 \mu \mathrm{m}$ C. succineum 61) Peridium pale brown, a textura intricata. Hairs hyphoid, irregularly branched, pale brown. Ascospores fusiform, $14-16.5 \times 5-7 \mu \mathrm{m}$ C. nepalense (Udagawa \& Y. Sugiy. 1982) Arx 61*) Peridial wall darker, with a different textura. Ascospores ellipsoidal with attenuated ends. Hairs brown ..............62 62) Textura intricata to epidermoidea. Ascomatal hairs flexuous, undulate or apically circinate. Ascospores $13-17 \times 7-$ $9 \mu \mathrm{m}$ C. murorum (and its relatives) 62*) Textura cephalothecoidea. Hairs straight or slightly curved, apically open circinate. Ascospores $15-17.5 \times 8.5-$ $10.5 \mu \mathrm{m}$ C. ancistrocladum 
63) (from 48) Ascomatal hairs undulate or coiled; ascospores ellipsoidal or ovate, occasionally irregular or bilaterally flattened

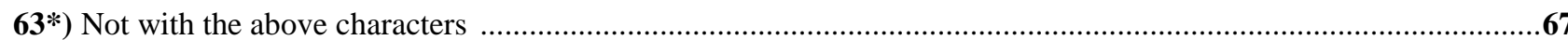

64) Ascomata dark in reflected light. Peridium of a textura intricata or epidermoidea. Ascomatal hairs undulate or spirally coiled. Ascospores ovoidal, 5-8 $\times 4-5 \mu \mathrm{m}$, bilaterally not flattened ............................................ C. nigricolor

64*) Not with the above characters

65) Ascospores ovoidal, bilaterally flattened, $8-11 \times 6-9 \mu \mathrm{m}$. Peridium of a textura angularis. Hairs undulate or much spirally coiled C. uniporum

65*) Ascospores not bilaterally flattened

. .66

66) Ascospores irregular in size and shape, $8-12 \times 5-6.5 \mu \mathrm{m}$. Peridium often of a textura cephalothecoidea. Ascomatal hairs horizontally expanding, 4-6 $\mu \mathrm{m}$ diam

C. repens

66*) Ascospores regular, ellipsoidal or ovate, 8.5-11 $\times 5-6.5 \mu \mathrm{m}$. Peridium of a textura angularis. Ascomatal hairs

2.5-4 $\mu \mathrm{m}$ broad, with long coils

C. spiralotrichum

67) Terminal hairs straight. Ascospores fusiform, ellipsoidal or reniform

.68

67*) Terminal hairs flexuous, arcuate or hyphoid, occasionally with a circinate tip; ascospores often fusiform ............70

68) Ascospores fusiform to broadly fusiform, 9.5-12 $\times 5-7 \mu \mathrm{m}$, with an apical or subapical germ pore. Colonies with abundant olive-green exudates .................................................... olivicolor K. Rodr. et al. in Rodriguez et al. 2002 68*) Ascospores different in shape. Colonies not described in the protologues or lacking exudates ...........................69

69) Lateral hairs arcuate. Ascospores ellipsoidal in frontal view, reniform or lunate in side view, 5-6.5 $\times 2.5-3 \times 2.5-3$ $\mu \mathrm{m}$, with an apical germ pore. Colonies without exudates

C. novae-caledonicum Udagawa et al. 1994

69*) Lateral hairs not arcuate. Ascospores reniform to slightly curved or sometimes with one flattened side, $8-13 \times$ 4.5-6 $\mu \mathrm{m}$, with one apical germ pore (rarely two). Exudates not described C. subcurvisporum Abdullah \& Al-Bader 1989

70) Ascospores reniform or lunate in frontal view. Hairs with copper or red encrustations

70*) Not with the above characters

71) Colonies with a pink exudate. Terminal hairs circinate, with 1-3 convolutions at the apex. Ascospores lunate, 8.5-

$10 \times 5-7 \mu \mathrm{m}$

C. lunasporium Udaiyan \& V.S. Hosag. 1991

N.B.: after reading the protologue, we think this taxon could be a later synonym of $C$. cupreum.

71*) Colonies with a red exudate. Terminal hairs circinate or coiled. Ascospores reniform or lunate, 7-10 $\times 4.5-6 \mu \mathrm{m} .$.

C. cupreum

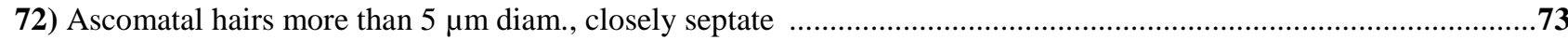

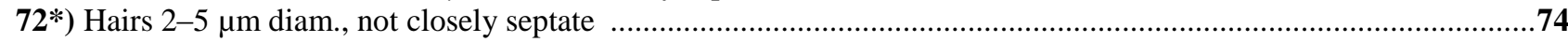

73) Ascomatal hairs 5-7 $\mu \mathrm{m}$ diam., arcuate, circinate or coiled at the apex. Ascospores ellipsoidal, 8.5-10.5 ×5-6 $\mu \mathrm{m}$..

C. lentum

73*) Ascomatal hairs 6-9 $\mu \mathrm{m}$ diam., arcuate, apically incurved or circinate. Ascospores ellipsoidal, often asymmetrical, 9-11 × 7-9 $\mu \mathrm{m}$, occasionally with a single germ pore, usually with two ......................................... turgidopilosum

74) Ascomatal hairs flexuous, thick-walled, smooth; ascospores $8-9.5 \times 5.5-6.5 \mu \mathrm{m}$, with a distinct germ pore

C. cymbiforme

74*) Not with the above characters

.75

75) Ascomatal hairs flexuous, apically undulate or curved, thin, verrucose. Peridium of a textura angularis. Ascospores 9-11 × 5-5.7 $\mu \mathrm{m}$, with a small germ pore ................................................................................ C. barilochense

75*) Not with the above characters . .76

76) (from 14 and 75) Peridium of a textura angularis. Ascospores with a nearly apical or slightly subapical germ pore ...

76*) Peridium with an outer layer of textura intricata. Ascospores with a lateral or distinctly subapical germ pore 77

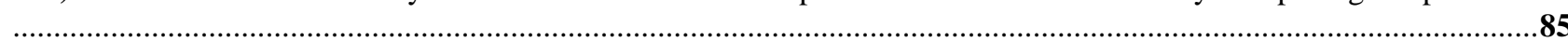

77) Hairs seta-like, straight, dark in reflected light. Ascospores fusiform to elongated pyriform, 9-11 ×4-6 $\mu \mathrm{m}$

C. atrobrunneum

77*) Hairs not seta-like, not black in reflected light

78) Colonies with an olive-green exudate. Terminal hairs straight, unbranched. Ascospores fusiform to broadly fusiform, 9.5-12 × 5-7 $\mu \mathrm{m}$............................................................. olivicolor K. Rodr. et al. in Rodriguez et al. 2002

78*) Colonies lacking exudates or with differently coloured exudates. Terminal hairs different

79) Colonies black. Ascomatal hairs undulate, rarely branched, constricted at septa and articulate. Ascospores broadly fusiform, 20-22 × 16-18 $\mu \mathrm{m}$, with a protuberant, subapical (sometimes apical) germ pore, 2-2.5 $\mu \mathrm{m}$ wide

C. sinaiense Mustafa \& Ezz-Eldin 1989

79*) Hairs not articulate, different in shape. Ascospores and germ pore smaller

80) Colonies dark. Ascomatal hairs pale, orange or ochraceous, arcuate or flexuous. Ascospores ellipsoidal or ovate, 7-

$9 \times 5-7 \mu \mathrm{m}$ C. lucknowense

80*) Colonies pale. Hairs darker, brown to black. Ascospores longer on average ................................................81

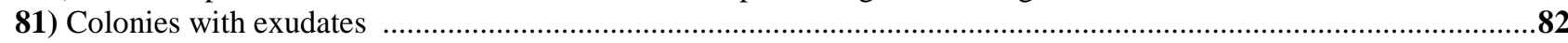

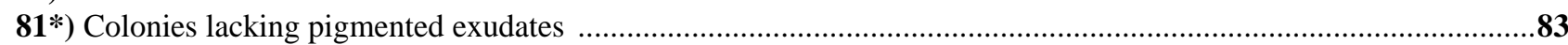

82) Exudates yellow-green. Ascomatal hairs arcuate, partly undulate. Ascospores ellipsoidal or broadly fusiform, 11-

$15 \times 6-8.5 \mu \mathrm{m}$, occasionally with two germ pores ............................................................................ gracile

82*) Exudates orange. Hairs arcuate or undulate, circinate. Ascospores ellipsoidal, often asymmetrical, 11-13 $\times 6-7$ 
83) Terminal hairs spirally coiled. Ascospores $10-11 \times 5.5-6.5 \mu \mathrm{m}$, inequilaterally fusiform to navicular

C. iranianum Asgari \& Zare 2011

$\mathbf{8 3}^{*}$ ) Terminal hairs arcuate or flexuous. Ascospores ellipsoidal-fusiform with (sub)acute ends, equilateral or slightly inequilateral at most

84) Ascospores $10-12.5 \times 6-7 \mu \mathrm{m}$. Hairs $4-6 \mu \mathrm{m}$ diam. near the base

84*) Ascospores 7-10 × 4.5-6 $\mu \mathrm{m}$. Hairs 2.5-4 (-5) $\mu \mathrm{m}$ near the base

C. muelleri

85) (from 76) Ascomatal hairs of two types: seta-like and dark or pale, thinner, branched. Ascospores ellipsoidalfusiform, $8-12 \times 5-7 \mu \mathrm{m}$...

C. cuniculorum

85*) Hairs unbranched, undulate or coiled

.86

86) Ascospores up to $11 \times 6.5 \mu \mathrm{m}$

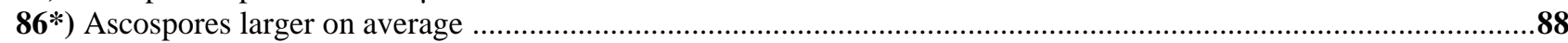

87) Ascospores $10-11 \times 5.5-6.5 \mu \mathrm{m}$, inequilaterally fusiform to navicular. Terminal hairs spirally coiled

C. iranianum Asgari \& Zare 2011

87*) Ascospores ovoidal or broadly fusiform, $8-11 \times 5-6.5 \mu \mathrm{m}$. Ascomatal hairs of two types: long, flexuous, apically circinate or coiled; short, tapering, often arranged in brushes

C. gelasinosporum

88) Ascospores $12-15 \times 7.5-9.5 \mu \mathrm{m}$, ellipsoidal. Terninal hairs coiled. Aleurioconidia often present ......C. gangligerum

88*) Ascospores narrower on average. Aleurioconidia absent

. .89

89) Colonies with orange exudates. Ascomata black in reflected light, with much coiled hairs. Ascospores fusiform or narrowly ovate, $10-12.5 \times 6.5-8 \mu \mathrm{m}$ C. jabalpurense

89*) Orange exudates absent. Ascomata not black in reflected light

.. .90

90) Colonies lacking exudates. Hairs undulate or spirally coiled. Ascospores fusiform or ovate, $12.5-14 \times 6.5-7.5 \mu \mathrm{m}$..

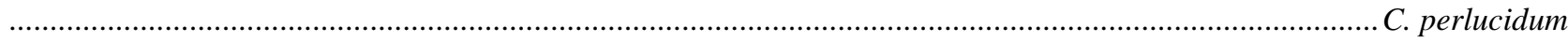

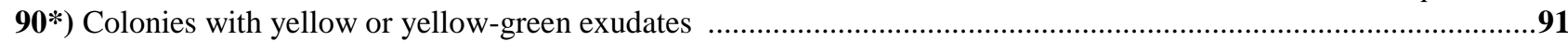

91) Ascospores fusiform or narrowly ovate, $10-13 \times 6-7 \mu \mathrm{m}$. Ascomatal hairs flexuous, undulate or coiled ......... C. raii

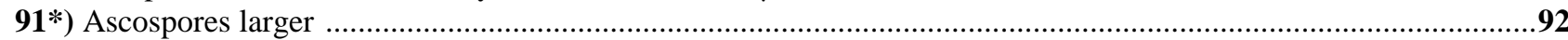

92) Ascomatal hairs spirally coiled, brown. Ascospores fusiform, $14-17 \times 7-8 \mu \mathrm{m}$................................... C. fusisporum

92*) Hairs flexuous or undulate, pale, thin. Ascospores elongate ellipsoidal or fusiform, $14-19 \times 6-8 \mu \mathrm{m}$ C. jodhpurense

93) (from 13) Ascospores fusiform, 22-27 × 8-9 $\mu \mathrm{m}$, without distinct germ pores. Ascomatal hairs straight or flexuous.

Aleurioconidia present

C. piluliferoides

93*) Ascospores at least partly with two germ pores

94) Ascospores with two subapical germ pores, ellipsoidal-fusiform, $17-21 \times 10-12 \mu \mathrm{m}$. Peridium of a textura intricata or epidermoidea. Hairs straight or arcuate ........................................................................... C. retardatum

94*) Ascospores with apical germ pores

. .95

95) Ascomata and ascomatal hairs pinkish-purple, the latter hyphoid, pale brown, unbranched. Ascospores broadly fusiform to musiform, 15.5-21 ×9-11.5 $\mu \mathrm{m}$, with a wall sometimes darkened around the pores

95*) Ascomata and ascomatal hairs not pinkish-purple

C. purpurascens (Udagawa \& Y. Sugiy. 1982) Arx

96) Ascospores fusiform or navicular, $18-23 \times 8-13 \mu \mathrm{m}$. Hairs flexuous and tapering. Colonies often with red exudates

96*) Ascospores smaller

C. megasporum

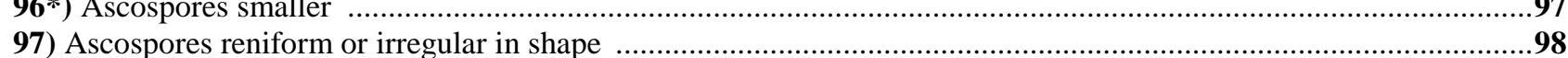

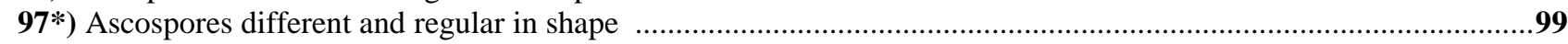

98) Ascospores reniform to slightly curved or sometimes with one flattened side, $8-13 \times 4.5-6 \mu$ m, with one apical germ pore (rarely two). Terminal hairs straight, unbranched .................. C. subcurvisporum Abdullah \& Al-Bader 1989 98*) Ascospores ellipsoidal or reniform, often irregular in shape, 10-13 $\times 7-9 \mu \mathrm{m}$, with two germ pores. Hairs flexuous

99) Ascospores fusiform, $15-18 \times 5-8.5 \mu \mathrm{m}$

C. variosporum

99*) Ascospores shorter

100

100) Hairs spirally coiled. Ascospores symmetrical, $15-18 \times 6.5-8 \mu \mathrm{m}$, darkened around the

100*) Hairs not spirally coiled

ticum

101) Terminal hairs flexuous or undulate. Ascospores often asymmetrical, $15-18 \times 7.5-8.5 \mu \mathrm{m}$

..................................................................................................................... . biporatum Cano \& Guarro 1987

101*) Terminal hairs straight, flexuous or undulate. Ascospores usually symmetrical, 13-19 × 5-7.5 $\mu \mathrm{m}$.... C. fusiforme 102) Ascomatal hairs 6-9 4 m diam., arcuate, apically incurved or circinate. Ascospores ellipsoidal, often asymmetrical, 9-11 $\times 7-9 \mu \mathrm{m}$, occasionally with a single germ pore C. turgidopilosum

102*) Ascomatal hairs $2-5 \mu \mathrm{m}$ diam ..... .103

103) Colonies usually with red exudates. Terminal hairs arcuate, apically circinate or coiled ...................................104

$\mathbf{1 0 3}^{*}$ ) Colonies with orange or yellow-green exudates. Hairs also of other types..................................................105

104) Hairs olivaceous green in reflected light. Ascospores inequilaterally fusiform or navicular, $8-12 \times 4-7 \mu \mathrm{m}$ 
104*) Hairs red or orange-red in reflected light. Ascospores fusiform or slightly inequilaterally fusiform, 11.1-13.5 $\times$ $4.3-5 \mu \mathrm{m}$ C. siamense Pornsuriya \& Soytong in Ponsuriya et al. 2011 105) Colonies with orange exudates. Hairs arcuate, apically circinate. Ascospores broadly fusiform or navicular, often asymmetrical, $11-14 \times 7-9 \mu \mathrm{m}$ C. flavigenum

105*) Colonies with yellow-green exudates .106

106) Ascomata spherical. Hairs arcuate, partly undulate, with a swollen basal cell. Ascospores ellipsoidal or broadly fusiform, $11-15 \times 6-8.5 \mu \mathrm{m}$, usually with a single germ pore, occasionally with two C. gracile 106*) Ascomata ampulliform. Hairs straight, seta-like. Ascospores ellipsoidal-fusiform, often asymmetrical, $9-14 \times$ $5.5-8 \mu \mathrm{m}$, occasionally with a single germ pore

C. virescens N.B.: C. thielavioideum K.T. Chen has been regarded as a synonym of C. virescens by Udagawa (1980), as a variety of the latter by Cannon (1986). According to von Arx et al. (1986) it differs from C. virescens in having larger ascomata with distinct hairs and ascospores with a single germ pore. 107) (from 5) Thermophilic. Ascomatal hairs flexuous, dichotomously or irregularly branched. Ascospores globose in frontal view, broadly ovoidal in side view, 7-9.5 ×6-8.5 $\times 4-6 \mu \mathrm{m}$, with an apical germ pore .............C. thermophilum 107*) Mesophilic, occasionally thermotolerant or psychrotolerant ..................................................................108 108) Ascospores broadly ovoidal or irregular, often inequilateral, 13-18 × 10-14 ×9-12 $\mu \mathrm{m}$, with an apical germ pore. Ascomatal hairs undulate C. uniapiculatum

108*) Ascospores smaller 109

109) Ascospores lacrymiform, bilaterally flattened, $9-11 \times 7-8 \times 6-7 \mu \mathrm{m}$, with a subapical or nearly apical germ pore. Terminal hairs flexuous or undulate, often branched C. senegalense 109*) Ascospores different in shape 110 110) Ascospores (sub)globose to broadly ovoidal, sometimes with a subapical or lateral germ pore. Hairs spirally coiled

110*) Ascospores different in shape, with an apical germ pore. Ascomatal hairs hyphoid, often branched

111) Ascospores often globose or ovoidal, bilaterally flattened, irregular in shape, $10-13 \times 9-10 \mu \mathrm{m}$, with a subapical or lateral germ pore. Terminal hairs unbranched or very rarely branched, with up to 20 turns

111*) Ascospores smaller, usually regular in shape, with an apical germ pore

112) Colonies restricted, with yellow exudates. Ascospores broadly ovoidal, often biapiculate, bilaterally flattened, $7-$ $11 \times 6-9 \times 4-6.5 \mu \mathrm{m}$ C. crispatum

112*) Colonies expanding, lacking yellow exudates. Ascospores slightly smaller

113) Hairs 5-8 $\mu \mathrm{m}$ diam., coiled and with coiled branchs. Ascospores broadly ovoidal, 7-8.5 × 6-7 × 5-6 $\mu \mathrm{m}$

C. medusarum

113*) Ascomatal hairs $2.5-5 \mu \mathrm{m}$ broad, unbranched

114) Ascomata with a dark apical area. Ascospores subglobose-ellipsoidal, rarely ovoidal, bilaterally flattened, $5.5-9 \times$ $5-7.5 \times 4.5-5.5 \mu \mathrm{m}$ C. acropullum X.W. Wang in Wang \& Zheng 2005

114*) Ascomata without a darker apical area. Ascospores broadly ovoidal

.115

115) Ascospores $8-9.5 \times 7-8 \times 6-7 \mu \mathrm{m}$. Ascomatal hairs with numerous coils

C. mollicellum

115*) Ascospores 7-8.5 $\times 6-7 \times 5-6 \mu \mathrm{m}$. Hairs flexuous, undulate or with 3-5 coils

C. brasiliense

116) Colonies sometimes with yellow-brown or orange-brown exudates. Peridium dark brown, a textura angularis covered with an outer layer of textura intricata. Hairs about $1 \mu \mathrm{m}$ diam. Asci cylindrical to clavate. Ascospores limoniform, bilaterally flattened, biapiculate, $8.5-10 \times 6.5-7.5 \times 5-6 \mu \mathrm{m}$. Scytalidium-like resting ascospores sometimes produced

C. luteum (J.N. Rai \& J.P. Tewari 1964) P.F. Cannon 116*) Colonies often with orange exudates. Peridium sulphur-yellow in reflected light, of a textura intricata tending to epidermoidea. Hairs up to $3 \mu \mathrm{m}$ diam. Asci cylindrical. Ascospores broadly fusiform to rhomboid, sometimes slightly inequilateral, 11-13 × 6-7.5 $\mu \mathrm{m}$. Anamorph Sporothrix-like

C. strumarium (J.N. Rai et al. 1964) P.F. Cannon 117) (from 1) Peridium of a textura angularis, almost glabrous or with some tapering hairs. Ascospores $10-12 \times 7-8.5$ $\times 5-6 \mu \mathrm{m}$, not dextrinoid. Conidia absent C. microascoides 117*) Peridium cephalothecoid with dark seta-like hairs. Ascospores 9-12 × 5-7 × 4-5 $\mu$ m, dextrinoid when young. Conidia present.

C. trigonosporum

N.B.: 1) C. cari-carvi A. Pande \& V.G. Rao (1990) has been omitted from the key, since the illustration of the ascospores in the protologue do not agree with the meagre description.

2) Where colony/cultural characteristics are mentioned they are from descriptions of growth on cornmeal agar usually at $28^{\circ}$ in the dark (von Arx et al. 1986).

\section{Acknowledgements}

The author thanks all friends who provided him with part of the material subject of this work. He is particularly indebted to A. Bizzi, C. Lavorato, L. Levorato and G. Robich. 


\section{References}

Abdullah SK, Al-Bader SM. 1989 - A new thermotolerant species of Chaetomium from Iraqi forest soil. International Journal of Mycology and Lichenology 4, 83-91.

Abdullah SK, Zora SE. 1993 - Chaetomium mesopotamicum, a new thermophilic species from Iraqi soil. Cryptogamic Botany 3, 387-389.

Ahmad S, Sultana K. 1973 - Contributions to the fungi of West Pakistan XVI. Biologia 19, 1-26.

Ames LM. 1949 - New cellulose destroying fungi isolated from military material and equipment. Mycologia 41, 637-648. http://dx.doi.org/10.2307/3755020

Ames L.M., 1963. A monograph of the Chaetomiaceae. United States Army Research and Development series 2, 1-125.

Asgari B, Zare R. 2011 - The genus Chaetomium in Iran, a phylogenetic study including six new species. Mycologia 103, 863-882. http://dx.doi.org/10.3852/10-349

Bainier G. 1909 - Monographie des Chaetomium et des Chaetomidium. Bulletin de la Société Mycologique de France 25, 191-237.

Barrasa JM. 1985 - Contribución al estudio taxonómico, ecológico y corológico de los ascomycetes coprófilos en España. Universidad de Alcalá de Henares (unpublished degree thesis).

Bell A. 2005 - An illustrated guide to the coprophilous ascomycetes of Australia. CBS Biodiversity Series 3, 1-172.

Beyer W. 1999 - Ergänzungen zur Pilzflora von Bayreuth und Umgebung. Teil 2. Zeitschrift für Mykologie 65, 41-80.

Bokhary HA, Parvez S. 1986 - Coprophilous fungi of Saudi Arabia 3. Coprophilous fungi of cow and rabbit dung. Proceedings of Saudi Biological Society 9, 13-21.

Calviello BO. 1971 - Estudio de las especies Argentinas del género Chaetomium. Revista del Museo Argentino de Ciencias Naturales Bernardino Rivadavia 3, 337-369.

Cannon PF. 1986 - A revision of Achaetomium, Achaetomiella and Subramaniula, and some similar species of Chaetomium. Transactions of the British Mycological Society 87, 45-76. http://dx.doi.org/10.1016/S0007-1536(86)80004-3

Cano J, Guarro J. 1987 - Soil ascomycetes from Spain XII. Chaetomium biporatum sp. nov. Nova Hedwigia 44, 543-546.

Caretta G, Piontelli E. 1996 - Coprophilous fungi from confined deers in Pavia (Lombardia, Italy). Boletín Micológico 11, 41-50.

Caretta G, Mangiarotti AM, Piontelli E. 1994 - Coprophilous fungi on horse, goat and sheep dung from Lombardia (Italy). Micologia Italiana 23, 11-20.

Caretta G, Piontelli E, Savino E, Bulgheroni A. 1998 - Some coprophilous fungi from Kenya. Mycopathologia 142, 125-134. http://dx.doi.org/10.1023/A:1006995313657

Carris LM, Glawe D. 1987 - Chaetomium histoplasmoides, a new species isolated from cysts of Heterodera glycines in Illinois. Mycotaxon 29, 383-391.

Carter A, Khan RS. 1982 - New and interesting Chaetomium species from East Africa. Canadian Journal of Botany 60, 1253-1262. http://dx.doi.org/10.1139/b82-159

Chandra S. 1964 - Chaetomium succineum Ames, a new record to India. Current Science 17, 532 533.

Chang JH, Wang YZ. 2009 - Six new records of ascomycetes from Taiwan. Fungal Science 24, 29-37.

Decock C, Hennebert GL. 1997 - A new species of Chaetomium from Ecuador. Mycological Research 101, 309-310. http://dx.doi.org/10.1017/S0953756296002456

Doveri F. 2004 - Fungi fimicoli italici. A.M.B. Fondazione Studi Micologici, Trento.

Doveri F. 2008 - Aggiornamento sul genere Chaetomium con descrizione di alcune specie coprofile, nuove per l'Italia - An update on the genus Chaetomium with descriptions of some coprophilous species, new to Italy. Pagine di Micologia 29, 1-60. 
Doveri F. 2011 - Additions to "Fungi Fimicoli Italici". An update on the occurrence of coprophilous basidiomycetes and ascomycetes in Italy with new records and descriptions. Mycosphere 2, 331-427.

Doveri F, Cacialli G, Caroti V. 2000 - A guide to the classification of fimicolous pyrenomycetes ss. lato from Italy. Contribution to the study of fimicolous fungi - XXXIII. In Micologia 2000. A.M.B. Fondazione Centro Studi Micologici, pp. 603-705.

Elshafie AE. 2005 - Coprophilous mycobiota of Oman. Mycotaxon 93, 355-357.

Eriksson OE. 1992 - The non-lichenized pyrenomycetes of Sweden. SBT, Lund.

Favre A. 2008 - Champignons coprophiles de la réserve naturelle du delta de la Dranse. Bulletin Mycologique et Botanique Dauphiné-Savoie 191, 23-28.

Gené J, Guarro J. 1996 - A new Chaetomium from Thailand. Mycological Research 100, 10051009. http://dx.doi.org/10.1016/S0953-7562(96)80055-4

Horie Y, Udagawa S. 1990 - New or interesting Chaetomium species from herbal drugs. Transactions of the Mycological Society of Japan 31, 249-258.

Hubálek Z. 1974 - Dispersal of fungi of the family Chaetomiaceae by free-living birds. I. A survey of records. Česká Mykologie 28, 65-79.

Hubka V, Mencl K, Skorepova M, Lyskova P, Zalabska E. 2011 - Phaeohyphomycosis and onychomycosis due to Chaetomium spp., including the first report of Chaetomium brasiliense infection. Medical Mycology 49, 724-733. http://dx.doi.org/10.3109/13693786.2011.572299

Karsten PA. 1888 - Symbolae ad Mycologiam Fennicam. XXVII. Meddelanden af Societas pro Fauna et Flora Fennica 16, 33-36.

Khumkomkhet P, Kanokmedhakul S, Kanokmedhakul K, Hahnvajanawong C, Soytong K. 2009 Antimalarial and cytotoxic depsidones from the fungus Chaetomium brasiliense. Journal of Natatural Products 72, 1487-1491. http://dx.doi.org/10.1021/np9003189

Li GY, Li BG, Yang T, Liu GY, Zhang GL. 2008 - Secondary metabolites from the fungus Chaetomium brasiliense. Helvetica Chimica Acta 91, 124-129. http://dx.doi.org/10.1002/hlca.200890002

Liou SC, Chen ZC. 1979 - Preliminary studies on coprophilous pyrenomycetes from Taiwan. Taiwania 24, 11-21.

Lodha BC. 1964 - Studies on coprophilous fungi. Journal of the Indian Botanical Society 43, 121140.

Lorenzo LE. 1993 - Especies coprófilas de la familia Chaetomiaceae en la Patagonia (Argentina). Boletín de la Sociedad Argentina de Botánica 29, 159-172.

Massee G, Salmon ES. 1901 - Researches on coprophilous fungi. Annals of Botany 15, 313-357.

Massee G, Salmon ES. 1902 - Researches on coprophilous fungi. II. Annals of Botany 16, 57-93.

Matsushima T. 1975 - Icones microfungorum a Matsushima lectorum. Published by the Author, Kobe, Japan.

Meyer JA, Lanneau C. 1967 - Deux nouvelles espèces de Chaetomium des sols africains. Bulletin de la Société Mycologique de France 83, 318-323.

Millner PD. 1975 - Ascomycetes of Pakistan: Chaetomium. Biologia 21, 39-73.

Mirza JH, Nasir MA. 1968 - Additions to the coprophilous fungi of West Pakistan. II. Nova Hedwigia 16, 283-288.

Moustafa AF, Ess El-Din EK. 1989 - Chaetomium sinaiense sp. nov., a new soil ascomycete from Egypt. Canadian Journal of Botany 67, 3417-3419. http://dx.doi.org/10.1139/b89-416

Oh H, Swenson DC, Gloer JB, Wicklow DT, Dowd PF. 1998 - Chaetochalasin A: a new bioactive metabolite from Chaetomium brasiliense. Tetrahedron Letters 39, 7633-7636.

Palliser HL. 1910 - Chaetomiaceae in: North American Flora 3 (1). The New York Botanical Garden 59-64.

Pande A, Rao VG. 1990 - Ascomycetes of western India-XIV. Journal of Economic and Taxonomic Botany 14, 157-161. 
Piontelli E, Rodrigo Cruz L, Alicia Toro Santa Maria M. 2006 - Coprophilous fungal community of wild rabbit in a park of a hospital (Chile): a taxonomic approach. Boletín Micológico 21, $1-17$.

Pornsuriya C, Lin FC, Kanokmedhakul S, Soytong K. 2008 - New record of Chaetomium species isolated from soil under pineapple plantation in Thailand. Journal of Agricultural Technology 4, 91-103.

Pornsuriya C, Soytong K, Poeaim S, Kanokmedhakul S, Khumkomkhet P, Lin FC, Wang HK, Hyde KD. 2011 - Chaetomium siamense sp. nov., a soil isolate from Thailand, produces a new chaetoviridin, G. Mycotaxon 115, 19-27. http://dx.doi.org/10.5248/115.19

Rai JN, Tewari JP, Mukerji KG. 1964 - Achaetomium, a new genus of ascomycetes. Canadian Journal of Botany 42, 693-697. http://dx.doi.org/10.1139/b64-064

Richardson MJ. 2001 - Diversity and occurrence of coprophilous fungi. Mycological Research 105, 387-402 http://dx.doi.org/10.1017/S0953756201003884

Richardson MJ. 2004 - Coprophilous fungi from Morocco. Botanical Journal of Scotland 56, 147162. http://dx.doi.org/10.1080/03746600408685075

Richardson MJ, Watling R. 1997 - Keys to fungi on dung. British Mycological Society.

Rodriguez K, Stchigel A, Guarro J. 2002 - Three new species of Chaetomium from soil. Mycologia 94, 116-126. http://dx.doi.org/10.2307/3761851

Saxena AS, Mukerji KG. 1973 - Fungi of Delhi. XVI. Further additions to Indian species of Chaetomium. Česká Mykologie 27, 162-164.

Sergejeva KS. 1961 - Species novae generis Chaetomium. III. Notulae Systematicae e Sectione Cryptogamica Instituti Botanici Komarovii Academiae Scientiarum U.R.S.S. 14, 139-150.

Seth HK. 1968 - Coprophilic Ascomycota from Germany. Nova Hedwigia 16, 495-499.

Seth H.K. 1970 - A monograph of the genus Chaetomium. Nova Hedwigia, suppl. 37, 1-133.

Sibounnavong P, Sibounnavong PS, Kanokmedhakul S, Soytong K. 2012 - Antifungal activities of Chaetomium brasilense $\mathrm{CB} 01$ and Chaetomium cupreum $\mathrm{CC} 03$ against $F$. oxysporum $\mathrm{f}$. sp. lycopersici race 2. Journal of Agricultural Technology 8, 1029-1038.

Singh SS, Chanda Jain R. 1969 - Chaetomiaceae of Chhatarpur. Indian Phytopathology 22, 393394.

Skolko AJ, Groves JW. 1953 - Notes on seed-borne Fungi. VII. Chaetomium. Canadian Journal of Botany 31, 779-809. http://dx.doi.org/10.1139/b53-060

Stratton R. 1921 - The Fimetariales of Ohio. Ohio Biological Survey 3 (2), n 12, 75-144.

Tóth S. 1963 - Data to the knowledge on the coprophilous microscopic fungi in Hungary I. Annales Historico-Naturales Musei Nationalis Hungarici Pars Botanica 55, 181-185.

Tóth S. 1965 - Data to the knowledge on the coprophilous microscopic fungi in Hungary II. Annales Historico-Naturales Musei Nationalis Hungarici Pars Botanica 57, 149-157.

Tóth S. 1967 - Data to the knowledge of the coprophilous microscopic fungi in Hungary III. Annales Historico-Naturales Musei Nationalis Hungarici Pars Botanica 59, 117-123.

Udagawa S. 1960 - A taxonomic study on the Japanese species of Chaetomium. Journal of General and Applied Microbiology 6, 223-251. http://dx.doi.org/10.2323/jgam.6.223

Udagawa S. - 1980: New or noteworthy ascomycetes from Southeast Asian soil I. Transactions of the Mycological Society of Japan 21, 17-34.

Udagawa S, Sugiyama Y. 1982 - New records and new species of ascomycetous microfungi from Nepal, a preliminary report on the expedition of 1980. Reports on the Cryptogamic Study in Nepal, March 1982 (Miscellaneous Publications of the Natural Science Museum, Tokyo), $11-46$.

Udagawa S, Toyazaki N, Yaguchi Y. 1997 - A new species of Chaetomium from house dust. Mycoscience 38, 399-402. http://dx.doi.org/10.1007/BF02461679

Udagawa S, Uchiyama S, Kamiya S. 1994 - Two new species of pyrenomycetous ascomycetes from New Caledonia. Mycoscience 35, 319-325. http://dx.doi.org/10.1007/BF02268501

Udaiyan K, Hosagoudar VS. 1991 - Some interesting fungi from the industrial water cooling towers of Madras - II. Journal of Economic and Taxonomic Botany 15, 649-666. 
Valldosera M. 1991 - Contribución al estudio de los hongos coprófilos - Ascomycotina - en España. Universitat Autonoma de Barcelona (unpublished degree thesis).

von Arx JA, Guarro J, Figueras MJ. 1986 - The ascomycete genus Chaetomium. Nova Hedwigia $84,1-162$.

Wang X, Zheng R. 2005 - Chaetomium ampulliellum sp. nov. (Chaetomiaceae, Ascomycota) and similar species from China. Nova Hedwigia 81, 247-255. http://dx.doi.org/10.1127/00295035/2005/0081-0247 